\title{
BOUNDEDNESS CRITERION FOR BILINEAR FOURIER MULTIPLIER OPERATORS
}

\author{
AKIHIKO MiYACHI AND NAOHITO TOMITA
}

(Received February 18, 2013)

\begin{abstract}
Bilinear Fourier multiplier operators corresponding to multipliers that are singular at the origin are considered. New criterions on such multipliers to assure the boundedness of the corresponding operators from $L^{p} \times L^{q}$ to $L^{r}, 1 / p+1 / q=1 / r$, are given in the range $1<p, q \leq \infty, 2 / 3<r<\infty$.
\end{abstract}

1. Introduction. For $m \in L^{\infty}\left(\boldsymbol{R}^{2 n}\right)$, the bilinear Fourier multiplier operator $T_{m}$ is defined by

$$
T_{m}(f, g)(x)=\frac{1}{(2 \pi)^{2 n}} \int_{\boldsymbol{R}^{2 n}} e^{i x \cdot(\xi+\eta)} m(\xi, \eta) \widehat{f}(\xi) \widehat{g}(\eta) d \xi d \eta
$$

for $f, g \in \mathcal{S}\left(\boldsymbol{R}^{n}\right)$, where $x, \xi, \eta \in \boldsymbol{R}^{n}$. Nowadays, it is well known that if $m$ satisfies the condition

$$
\left|\partial_{\xi}^{\alpha} \partial_{\eta}^{\beta} m(\xi, \eta)\right| \leq C_{\alpha, \beta}(|\xi|+|\eta|)^{-(|\alpha|+|\beta|)}
$$

for sufficiently many multi-indices $\alpha, \beta \in N_{0}^{n}=\{0,1,2, \ldots\}^{n}$ then $T_{m}$ is bounded from $H^{p} \times H^{q}$ to $L^{r}, 0<p, q, r \leq \infty, 1 / p+1 / q=1 / r$, where $H^{p}$ and $H^{q}$ are Hardy spaces. (Throughout this paper, $H^{p}\left(\boldsymbol{R}^{n}\right), L^{r}\left(\boldsymbol{R}^{n}\right)$, etc. are often abbreviated to $H^{p}, L^{r}$, etc. if the base space $\boldsymbol{R}^{n}$ is obviously recognized from the context.) These facts were proved by Coifman-Meyer [3, 12], Kenig-Stein [11], Grafakos-Kalton [5], and Grafakos-Torres [9]. It is an interesting problem to find the conditions of type (1.1) with differentiability order as small as possible that assure the boundedness of $T_{m}$. The condition given in the papers $[3,12,11$, 5,9] is at least (1.1) for $|\alpha|+|\beta| \leq 2 n+1$ (although this order is only implicitly given in the papers). Recently, much more weak conditions were found by Tomita [19], Grafakos-Si [7], Grafakos-Miyachi-Tomita [6], and Miyachi-Tomita [13]. The purpose of the present paper is to give conditions different from those treated in $[19,7,6,13]$ and partly improve the results of these papers.

Before we give our results in detail, we shall recall the result of [13]. We shall write

$$
\left\|T_{m}\right\|_{H^{p}\left(\boldsymbol{R}^{n}\right) \times H^{q}\left(\boldsymbol{R}^{n}\right) \rightarrow L^{r}\left(\boldsymbol{R}^{n}\right)}
$$

to denote the smallest constant $C$ satisfying

$$
\left\|T_{m}(f, g)\right\|_{L^{r}\left(\boldsymbol{R}^{n}\right)} \leq C\|f\|_{H^{p}\left(\boldsymbol{R}^{n}\right)}\|g\|_{H^{q}\left(\boldsymbol{R}^{n}\right)}
$$

2010 Mathematics Subject Classification. Primary 42B15; Secondary 42B20.

Key words and phrases. Bilinear Fourier multipliers, Hörmander multiplier theorem, Littlewood-Paley theory. 
for all $f \in \mathcal{S}\left(\boldsymbol{R}^{n}\right) \cap H^{p}\left(\boldsymbol{R}^{n}\right)$ and $g \in \mathcal{S}\left(\boldsymbol{R}^{n}\right) \cap H^{q}\left(\boldsymbol{R}^{n}\right)$. We define

$$
\left\|T_{m}\right\|_{L^{\infty}\left(\boldsymbol{R}^{n}\right) \times L^{\infty}\left(\boldsymbol{R}^{n}\right) \rightarrow B M O\left(\boldsymbol{R}^{n}\right)}
$$

in the same way by replacing the norms $\|\cdot\|_{H^{p}},\|\cdot\|_{H^{q}},\|\cdot\|_{L^{r}}$ by $\|\cdot\|_{L^{\infty}},\|\cdot\|_{L^{\infty}},\|\cdot\|_{B M O}$, respectively. We use the convention that $H^{p}=L^{p}$ for $1<p \leq \infty$. In [13], we used the product type Sobolev norm, which is defined as follows. For $s_{1}, s_{2} \in \boldsymbol{R}$ and for $F \in \mathcal{S}^{\prime}\left(\boldsymbol{R}^{2 n}\right)$, we define

$$
\|F\|_{W^{\left(s_{1}, s_{2}\right)}\left(\boldsymbol{R}^{2 n}\right)}=\left\|\left(I-\Delta_{\xi}\right)^{s_{1} / 2}\left(I-\Delta_{\eta}\right)^{s_{2} / 2} F(\xi, \eta)\right\|_{L_{\xi, \eta}^{2}}
$$

where $\xi, \eta \in \boldsymbol{R}^{n}$ and

$$
\begin{aligned}
& \left(I-\Delta_{\xi}\right)^{s_{1} / 2}\left(I-\Delta_{\eta}\right)^{s_{2} / 2} F(\xi, \eta) \\
& \quad=\frac{1}{(2 \pi)^{2 n}} \int_{\boldsymbol{R}^{2 n}} e^{i(x \cdot \xi+y \cdot \eta)}\left(1+|x|^{2}\right)^{s_{1} / 2}\left(1+|y|^{2}\right)^{s_{2} / 2} \widehat{F}(x, y) d x d y
\end{aligned}
$$

For $m \in L^{\infty}\left(\boldsymbol{R}^{2 n}\right)$ and $j \in \boldsymbol{Z}$, we set

$$
m_{j}(\xi, \eta)=m\left(2^{j} \xi, 2^{j} \eta\right) \Psi(\xi, \eta), \quad(\xi, \eta) \in \boldsymbol{R}^{n} \times \boldsymbol{R}^{n},
$$

where $\Psi$ is a function in $\mathcal{S}\left(\boldsymbol{R}^{2 n}\right)$ satisfying

$$
\operatorname{supp} \Psi \subset\left\{\zeta \in \boldsymbol{R}^{2 n} ; 1 / 2 \leq|\zeta| \leq 2\right\}, \quad \sum_{k \in \boldsymbol{Z}} \Psi\left(\zeta / 2^{k}\right)=1, \quad \zeta \in \boldsymbol{R}^{2 n} \backslash\{0\} .
$$

The result of [13] reads as follows.

Theorem A ([13]). Let $0<p, q, r \leq \infty$ and $1 / p+1 / q=1 / r$. If $s_{1}>$ $\max \{n / 2, n / p-n / 2\}, s_{2}>\max \{n / 2, n / q-n / 2\}$, and $s_{1}+s_{2}>n / r-n / 2$, then

$$
\left\|T_{m}\right\|_{H^{p}\left(\boldsymbol{R}^{n}\right) \times H^{q}\left(\boldsymbol{R}^{n}\right) \rightarrow L^{r}\left(\boldsymbol{R}^{n}\right)} \sup _{j \in \boldsymbol{Z}}\left\|m_{j}\right\|_{W^{\left(s_{1}, s_{2}\right)}\left(\boldsymbol{R}^{2 n}\right)},
$$

where $H^{\infty} \times H^{\infty} \rightarrow L^{\infty}$ is replaced by $L^{\infty} \times L^{\infty} \rightarrow B M O$ if $p=q=r=\infty$. Conversely, if (1.5) with the same replacement in the case $p=q=r=\infty$ holds, then $s_{1} \geq \max \{n / 2, n / p-n / 2\}, s_{2} \geq \max \{n / 2, n / q-n / 2\}$, and $s_{1}+s_{2} \geq n / r-n / 2$.

In particular, if $(1 / p, 1 / q)$ is in the domain

$$
I_{0}: 0 \leq 1 / p \leq 1, \quad 0 \leq 1 / q \leq 1, \quad 1 / p+1 / q \leq 3 / 2,
$$

then (1.5) holds for $s_{1}>n / 2$ and $s_{2}>n / 2$. As far as the boundedness in $H^{p} \times H^{q} \rightarrow L^{r}$ for $(1 / p, 1 / q) \in I_{0}$ is concerned, this result is the sharpest among the estimates given in $[19,7,6,13]$ (see Remark 1.3 given at the end of this section). The following figure shows the domain $I_{0}$. 


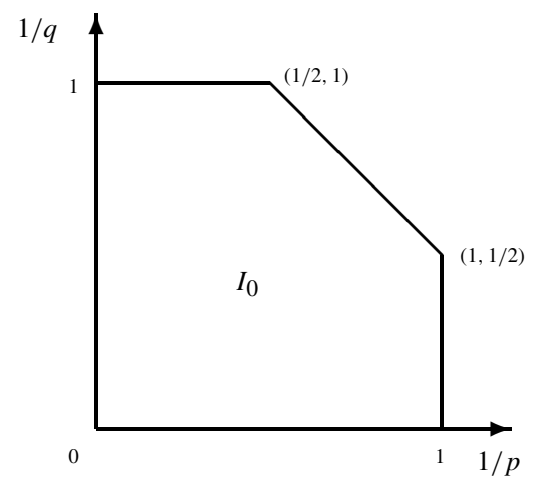

By the claim in the latter part of Theorem A, we cannot essentially relax the conditions of the theorem so far as we use the scale in the right-hand side of (1.5) to measure the smoothness of $m$. In the present paper, we shall introduce other scales to measure the smoothness of $m$ and give different criterions to assure the boundedness of $T_{m}$ for $(1 / p, 1 / q) \in I_{0}$.

The following is the first main result of this paper.

THEOREM 1.1. If $s>n / 2$, then

$$
\begin{aligned}
& \left\|T_{m}\right\|_{L^{2}\left(\boldsymbol{R}^{n}\right) \times L^{2}\left(\boldsymbol{R}^{n}\right) \rightarrow L^{1}\left(\boldsymbol{R}^{n}\right)} \\
& \quad \lesssim \sup _{j \in \boldsymbol{Z}}\left(\|\|\left(I-\Delta_{\xi}\right)^{s / 2} m_{j}(\xi, \eta)\left\|_{L_{\xi}^{2}}\right\|_{L_{\eta}^{\infty}}+\|\|\left(I-\Delta_{\eta}\right)^{s / 2} m_{j}(\xi, \eta)\left\|_{L_{\eta}^{2}}\right\|_{L_{\xi}^{\infty}}\right) .
\end{aligned}
$$

Since, for $s>n / 2$, the right-hand side of (1.6) is smaller than $\sup _{j \in \boldsymbol{Z}}\left\|m_{j}\right\|_{W^{(s, s)}\left(\boldsymbol{R}^{2 n)}\right.}$ by virtue of the Sobolev embedding, Theorem 1.1 is an improvement of the corresponding claim of Theorem A.

The second main result of this paper reads as follows.

THEOREM 1.2. Let $1 \leq p, q \leq \infty, 2 / 3 \leq r \leq \infty$, and $1 / p+1 / q=1 / r$. If

$$
s>s(p, q)=\max \{n / 2, n / p, n / q, n-n / r, n / r-n / 2\}
$$

and

$$
\begin{aligned}
1 / t & =t(p, q)^{-1}=s(p, q) / n-1 / 2 \\
& =\max \{0,1 / p-1 / 2,1 / q-1 / 2,1 / 2-1 / r, 1 / r-1\}
\end{aligned}
$$

then

$$
\left\|T_{m}\right\|_{H^{p}\left(\boldsymbol{R}^{n}\right) \times H^{q}\left(\boldsymbol{R}^{n}\right) \rightarrow L^{r}\left(\boldsymbol{R}^{n}\right)} \sup _{j \in \boldsymbol{Z}}\left\|m_{j}\right\|_{B_{t, \infty}^{s}\left(\boldsymbol{R}^{2 n}\right)},
$$

where $H^{\infty} \times H^{\infty} \rightarrow L^{\infty}$ is replaced by $L^{\infty} \times L^{\infty} \rightarrow$ BMO if $p=q=r=\infty$. Conversely, if $s>0$ and (1.7) with the same replacement in the case $p=q=r=\infty$ holds for some $t \in[1, \infty]$, then $s \geq s(p, q)$. 
See Section 2 for the definition of Besov spaces $B_{t, \infty}^{s}\left(\boldsymbol{R}^{2 n}\right)$. To clarify the values of $s(p, q)$ and $t(p, q)$ of this theorem, we divide the region $I_{0}$ into 5 regions $J_{0}, \ldots, J_{4}$ as follows:

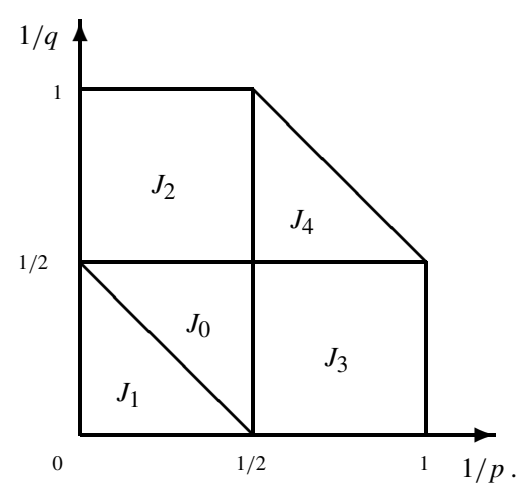

Then

$$
\begin{aligned}
s(p, q)=\left\{\begin{array}{lll}
n / 2 & \text { if } \quad(1 / p, 1 / q) \in J_{0} ; \\
n-n / r & \text { if } \quad(1 / p, 1 / q) \in J_{1} ; \\
n / q & \text { if } \quad(1 / p, 1 / q) \in J_{2} ; \\
n / p & \text { if } \quad(1 / p, 1 / q) \in J_{3} ; \\
n / r-n / 2 & \text { if } \quad(1 / p, 1 / q) \in J_{4} ;
\end{array}\right. \\
t(p, q)^{-1}=\left\{\begin{array}{lll}
0 & \text { if } \quad(1 / p, 1 / q) \in J_{0} ; \\
1 / 2-1 / r & \text { if } \quad(1 / p, 1 / q) \in J_{1} ; \\
1 / q-1 / 2 & \text { if } \quad(1 / p, 1 / q) \in J_{2} ; \\
1 / p-1 / 2 & \text { if } \quad(1 / p, 1 / q) \in J_{3} ; \\
1 / r-1 & \text { if } \quad(1 / p, 1 / q) \in J_{4} ;
\end{array}\right.
\end{aligned}
$$

where $1 / p+1 / q=1 / r$.

Let us compare Theorem 1.2 with Theorem A. If $(1 / p, 1 / q) \in I_{0}$ and if $1 / p=1$ or $1 / q=1$ or $1 / r=0$ or $1 / r=3 / 2$, then $s(p, q)=n$ and $t(p, q)=2$. In these cases, we have

$$
\begin{aligned}
B_{t(p, q), \infty}^{s(p, q)+\varepsilon}\left(\boldsymbol{R}^{2 n}\right)=B_{2, \infty}^{n+\varepsilon}\left(\boldsymbol{R}^{2 n}\right) & \hookrightarrow B_{2,2}^{n+\varepsilon / 2}\left(\boldsymbol{R}^{2 n}\right)=W^{n+\varepsilon / 2}\left(\boldsymbol{R}^{2 n}\right) \\
& \hookrightarrow W^{(n / 2+\varepsilon / 4, n / 2+\varepsilon / 4)}\left(\boldsymbol{R}^{2 n}\right),
\end{aligned}
$$

and hence the claim of Theorem 1.2 is covered by Theorem A. For other $(1 / p, 1 / q) \in I_{0}$, i.e., for $0 \leq 1 / p, 1 / q<1$ and $0<1 / p+1 / q<3 / 2$, we have $n / 2 \leq s(p, q)<n$ and $2<t(p, q) \leq \infty$. In this case, Theorem 1.2 covers multipliers that cannot be dealt with by Theorem A. In particular, for $(1 / p, 1 / q) \in J_{0}$, Theorem 1.2 implies that $T_{m}$ is bounded in $L^{p} \times L^{q} \rightarrow L^{r}, 1 / p+1 / q=1 / r$, if $m$ satisfies (1.1) for $|\alpha|+|\beta| \leq[n / 2]+1$, which does 
not follow from Theorem A. The precise statement will be given in Proposition 5.1 in Section 5 .

Here we give a remark on the argument to prove Theorems 1.1 and 1.2. We shall prove the estimate (1.6) or (1.7) for $m \in \mathcal{S}\left(\boldsymbol{R}^{2 n}\right)$ or even for $m \in C_{0}^{\infty}\left(\boldsymbol{R}^{2 d}\right)$. Once this is proved, then a limiting argument will show that the estimate holds for more general $m$ with the righthand side finite. In the argument of the succeeding sections, we shall not mention explicitly that we are treating only multipliers $m$ in these good classes.

The contents of this paper are as follows. In Section 2, we recall some preliminary facts. In Sections 3 and 4, we prove Theorems 1.1 and 1.2 respectively. In Section 5, we give the proposition mentioned above. In Appendix, we shall observe that Theorem 1.2 can be directly applied to the first commutator of Calderón.

REMARK 1.3. Here we briefly review the results of $[19,7,6]$. Although these papers deal with multilinear operators, here we consider only the bilinear case. For $1<t<\infty$ and $s>0$ and for functions $F(\xi, \eta)$ on $\boldsymbol{R}^{2 n}$, we define the Sobolev norm $\|F\|_{W_{t}^{s}\left(\boldsymbol{R}^{2 n}\right)}$ by

$$
\|F\|_{W_{t}^{s}\left(\boldsymbol{R}^{2 n}\right)}=\left\|\left(I-\Delta_{\xi}-\Delta_{\eta}\right)^{s / 2} F(\xi, \eta)\right\|_{L^{t}\left(\boldsymbol{R}^{2 n}\right)},
$$

where

$$
\left(I-\Delta_{\xi}-\Delta_{\eta}\right)^{s / 2} F(\xi, \eta)=\frac{1}{(2 \pi)^{2 n}} \int_{\boldsymbol{R}^{2 n}} e^{i(x \cdot \xi+y \cdot \eta)}\left(1+|x|^{2}+|y|^{2}\right)^{s / 2} \widehat{F}(x, y) d x d y .
$$

In the case $t=2$, we simply write $W^{s}\left(\boldsymbol{R}^{2 n}\right)$ instead of $W_{t}^{s}\left(\boldsymbol{R}^{2 n}\right)$. We consider the estimate

$$
\left\|T_{m}\right\|_{L^{p} \times L^{q} \rightarrow L^{r}} \lesssim \sup _{j \in \boldsymbol{Z}}\left\|m_{j}\right\|_{X}, \quad 1 / p+1 / q=1 / r,
$$

for a function space $X$ on $\boldsymbol{R}^{2 n}$, where $m_{j}$ is defined by (1.3). Now, firstly, [19, Theorem 1.1] asserts that (1.8) holds with $X=W^{s}\left(\boldsymbol{R}^{2 n}\right)$ if $s>n$ and $1<p, q, r<\infty$. This result is covered by Theorem A since $W^{s}\left(\boldsymbol{R}^{2 n}\right) \hookrightarrow W^{(s / 2, s / 2)}\left(\boldsymbol{R}^{2 n}\right)$. Secondly, [7, Theorem 1.1] asserts that (1.8) holds with $X=W_{t}^{s}\left(\boldsymbol{R}^{2 n}\right)$ if $1<t \leq 2,2 n \geq s>2 n / t$, and

$$
1<p, q \leq \infty, \quad r<\infty, \quad(2 n-s) / p+s / q<s, \quad s / p+(2 n-s) / q<s .
$$

(In fact, this is not explicitly stated in [7, Theorem 1.1], but it follows from the proof given in [7, Section 3], combined with some additional arguments of duality and interpolation as given in [7, Proof of Corollary 1.1].) This result restricted to $(1 / p, 1 / q) \in I_{0}$ is covered by Theorem A since the Sobolev embedding gives

$$
W_{t}^{s}\left(\boldsymbol{R}^{2 n}\right) \hookrightarrow W^{s^{\prime}}\left(\boldsymbol{R}^{2 n}\right) \hookrightarrow W^{\left(s^{\prime} / 2, s^{\prime} / 2\right)}\left(\boldsymbol{R}^{2 n}\right)
$$

with $s^{\prime}=s-2 n / t+n>n$. Finally, [6, Theorem 1.1] is the same as Theorem A for $(p, q)=(2, \infty)$ or $(\infty, 2)$.

2. Preliminaries. For two nonnegative quantities $A$ and $B$, the notation $A \lesssim B$ means that $A \leq C B$ for some unspecified constant $C>0$, and $A \approx B$ means that $A \lesssim B$ and $B \lesssim A$. We denote by $\chi_{S}$ the characteristic function of a set $S$. For $1 \leq p \leq \infty, p^{\prime}$ is the conjugate exponent of $p$, that is, $1 / p+1 / p^{\prime}=1$. 
Let $\mathcal{S}\left(\boldsymbol{R}^{n}\right)$ and $\mathcal{S}^{\prime}\left(\boldsymbol{R}^{n}\right)$ be the Schwartz spaces of all rapidly decreasing smooth functions and tempered distributions, respectively. We define the Fourier transform $\mathcal{F} f$ and the inverse Fourier transform $\mathcal{F}^{-1} f$ of $f \in \mathcal{S}\left(\boldsymbol{R}^{n}\right)$ by

$$
\mathcal{F} f(\xi)=\widehat{f}(\xi)=\int_{\boldsymbol{R}^{n}} e^{-i x \cdot \xi} f(x) d x \quad \text { and } \quad \mathcal{F}^{-1} f(x)=\frac{1}{(2 \pi)^{n}} \int_{\boldsymbol{R}^{n}} e^{i x \cdot \xi} f(\xi) d \xi .
$$

For a function $\sigma(x, \xi) \in L^{\infty}\left(\boldsymbol{R}^{n} \times \boldsymbol{R}^{n}\right)$, we define the linear pseudo-differential operator $\sigma(X, D)$ by

$$
\sigma(X, D) f(x)=\frac{1}{(2 \pi)^{n}} \int_{\boldsymbol{R}^{n}} e^{i x \cdot \xi} \sigma(x, \xi) \widehat{f}(\xi) d \xi, \quad f \in \mathcal{S}\left(\boldsymbol{R}^{n}\right) .
$$

In particular, if $\sigma$ is an $x$-independent symbol, then we denote by $\sigma(D)$ the corresponding linear Fourier multiplier operator.

The Hardy-Littlewood maximal operator $M$ is defined by

$$
M f(x)=\sup _{r>0} \frac{1}{r^{n}} \int_{|x-y|<r}|f(y)| d y,
$$

where $f$ is a locally integrable function on $\boldsymbol{R}^{n}$. We also use the notation $M_{q} f(x)=$ $M\left(|f|^{q}\right)(x)^{1 / q}$.

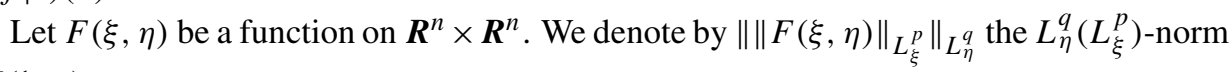
of $F(\xi, \eta)$,

$$
\|\| F(\xi, \eta)\left\|_{L_{\xi}^{p}}\right\|_{L_{\eta}^{q}}=\left\{\int_{\boldsymbol{R}^{n}}\left(\int_{\boldsymbol{R}^{n}}|F(\xi, \eta)|^{p} d \xi\right)^{q / p} d \eta\right\}^{1 / q}
$$

with usual modifications if $p=\infty$ or $q=\infty$. In the case $p=q$, we simply write $\|\cdot\|_{L_{\xi, \eta}^{p}}$ instead of \|\|$\cdot\left\|_{L_{\xi}^{p}}\right\|_{L_{\eta}^{q}}$.

Let $\Psi \in \mathcal{S}\left(\boldsymbol{R}^{n}\right)$ be as in (1.4) with $2 n$ replaced by $n$, and set $\Psi_{0}(\xi)=1-\sum_{k=1}^{\infty} \Psi\left(\xi / 2^{k}\right)$ and $\Psi_{k}(\xi)=\Psi\left(\xi / 2^{k}\right)$ if $k \geq 1$. Note that $\operatorname{supp} \Psi_{0} \subset\{|\xi| \leq 2\}$, supp $\Psi_{k} \subset\left\{2^{k-1} \leq|\xi| \leq\right.$ $\left.2^{k+1}\right\}$ if $k \geq 1$, and $\sum_{k=0}^{\infty} \Psi_{k}(\xi)=1$. For $1 \leq p, q \leq \infty$ and $s \in \boldsymbol{R}$, the Besov space $B_{p, q}^{s}\left(\boldsymbol{R}^{n}\right)$ consists of all $f \in \mathcal{S}^{\prime}\left(\boldsymbol{R}^{n}\right)$ such that

$$
\|f\|_{B_{p, q}^{s}}=\left(\sum_{k=0}^{\infty} 2^{k s q}\left\|\Psi_{k}(D) f\right\|_{L^{p}}^{q}\right)^{1 / q}=\left(\sum_{k=0}^{\infty} 2^{k s q}\left\|\left(\mathcal{F}^{-1} \Psi_{k}\right) * f\right\|_{L^{p}}^{q}\right)^{1 / q}<\infty .
$$

It is well known that $B_{2,2}^{s}\left(\boldsymbol{R}^{n}\right)=W^{s}\left(\boldsymbol{R}^{n}\right)$. See Triebel [20] for more details on Besov spaces.

Let $0<p \leq \infty$, and let $\phi \in \mathcal{S}\left(\boldsymbol{R}^{n}\right)$ be such that $\int_{\boldsymbol{R}^{n}} \phi(x) d x \neq 0$. Then the Hardy space $H^{p}\left(\boldsymbol{R}^{n}\right)$ consists of all $f \in \mathcal{S}^{\prime}\left(\boldsymbol{R}^{n}\right)$ such that

$$
\|f\|_{H^{p}}=\left\|\sup _{0<t<\infty}\left|\phi_{t} * f\right|\right\|_{L^{p}}<\infty
$$

where $\phi_{t}(x)=t^{-n} \phi(x / t)$. It is well known that $H^{p}\left(\boldsymbol{R}^{n}\right)=L^{p}\left(\boldsymbol{R}^{n}\right)$ if $1<p \leq \infty$. See Stein [17, Chapter 3] for more details on Hardy spaces.

Let $\phi_{0}$ be a $C^{\infty}$-function on $[0, \infty)$ satisfying

$$
\phi_{0}(t)=1 \quad \text { on } \quad[0,1 / 8], \quad \operatorname{supp} \phi_{0} \subset[0,1 / 4] .
$$


We set $\phi_{1}(t)=1-\phi_{0}(t)$, and define the functions $\Phi_{(i, j)}$ on $\boldsymbol{R}^{2 n} \backslash\{0\},(i, j) \in\{0,1\}^{2}$, by

$$
\Phi_{(i, j)}(\xi, \eta)=\phi_{i}\left(|\xi| / \sqrt{|\xi|^{2}+|\eta|^{2}}\right) \phi_{j}\left(|\eta| / \sqrt{|\xi|^{2}+|\eta|^{2}}\right),
$$

where $(\xi, \eta) \in \boldsymbol{R}^{n} \times \boldsymbol{R}^{n} \backslash\{(0,0)\}$. We note that $\Phi_{(0,0)}=0$, and have

Lemma 2.1 ([4, Lemma 3.1], [19, Section 5]). $\quad$ (1) For $(\xi, \eta) \neq(0,0)$,

$$
\Phi_{(1,1)}(\xi, \eta)+\Phi_{(0,1)}(\xi, \eta)+\Phi_{(1,0)}(\xi, \eta)=1 .
$$

(2) Each $\Phi_{(i, j)}$ satisfies

$$
\left|\partial_{\xi}^{\alpha} \partial_{\eta}^{\beta} \Phi_{(i, j)}(\xi, \eta)\right| \leq C_{(i, j)}^{\alpha, \beta}(|\xi|+|\eta|)^{-(|\alpha|+|\beta|)}
$$

for all multi-indices $\alpha, \beta$.

(3) $\operatorname{supp} \Phi_{(1,1)} \subset\{(\xi, \eta) ;|\xi| / 8 \leq|\eta| \leq 8|\xi|\}$, supp $\Phi_{(0,1)} \subset\{(\xi, \eta) ;|\xi| \leq|\eta| / 2\}$ and $\operatorname{supp} \Phi_{(1,0)} \subset\{(\xi, \eta) ;|\eta| \leq|\xi| / 2\}$.

The following fact with $s=0$ appears as [20, Proposition 1.3.2].

LEMMA 2.2 ([19, Lemma 3.3]). Let $2 \leq q \leq \infty, r>0$ and $s \geq 0$. If $\operatorname{supp} f \subset\{x \in$ $\left.\boldsymbol{R}^{n} ;|x| \leq r\right\}$, then

$$
\left(\int_{\boldsymbol{R}^{n}}\left|(1+|\xi|)^{s} \widehat{f}(\xi)\right|^{q} d \xi\right)^{1 / q} \lesssim\left(\int_{\boldsymbol{R}^{n}}\left|(I-\Delta)^{s / 2} f(x)\right|^{2} d x\right)^{1 / 2}
$$

where the implicit constant depends only on $n, q, r, s$.

LEMmA 2.3 ([20, Remark 2.8.2/1]). Let $1 \leq p, q \leq \infty$ and $s>0$. Then

$$
\|f g\|_{B_{p, q}^{s}} \lesssim\|f\|_{B_{p, q}^{s}}\|g\|_{B_{\infty, q}^{s}}
$$

for all $f \in B_{p, q}^{s}\left(\boldsymbol{R}^{n}\right)$ and all $g \in B_{\infty, q}^{s}\left(\boldsymbol{R}^{n}\right)$.

The fact in the next lemma is well known in the Littlewood-Paley theory for Hardy spaces. In fact, using the characterization of the Hardy space $H^{1}$ by Littlewood-Paley square functions ([20, 5.2.4]), we can give its proof.

Lemma 2.4. Let $A>1$. Then

$$
\left\|\sum_{j \in \boldsymbol{Z}} f_{j}\right\|_{H^{1}} \lesssim\left\|\left(\sum_{j \in \boldsymbol{Z}}\left|f_{j}\right|^{2}\right)^{1 / 2}\right\|_{L^{1}}
$$

for all sequences $\left\{f_{j}\right\}_{j \in \boldsymbol{Z}}$ satisfying supp $\widehat{f}_{j} \subset\left\{\xi \in \boldsymbol{R}^{n} ; A^{-1} 2^{j} \leq|\xi| \leq A 2^{j}\right\}$.

3. Proof of Theorem 1.1. In this section, we shall prove Theorem 1.1. The proof basically depends on the paraproduct argument. We first prepare several lemmas.

LEMMA 3.1. Let $s \in \boldsymbol{R}$, and let $\widetilde{\Psi} \in \mathcal{S}\left(\boldsymbol{R}^{2 n}\right)$ be such that supp $\widetilde{\Psi}$ is a compact subset of $\boldsymbol{R}^{2 n}$ away from the origin. Assume that $\Phi \in C^{\infty}\left(\boldsymbol{R}^{2 n} \backslash\{0\}\right)$ satisfies

$$
\left|\partial_{\xi}^{\alpha} \partial_{\eta}^{\beta} \Phi(\xi, \eta)\right| \leq C_{\alpha, \beta}(|\xi|+|\eta|)^{-|\alpha|-|\beta|}
$$


for all $\alpha, \beta \in N_{0}^{n}$. Set

$$
\widetilde{m}_{j}(\xi, \eta)=m\left(2^{j} \xi, 2^{j} \eta\right) \Phi\left(2^{j} \xi, 2^{j} \eta\right) \widetilde{\Psi}(\xi, \eta) .
$$

Then

$$
\begin{aligned}
& \sup _{j \in \boldsymbol{Z}}\|\|\left(I-\Delta_{\xi}\right)^{s / 2} \tilde{m}_{j}(\xi, \eta)\left\|_{L_{\xi}^{2}}\right\|_{L_{\eta}^{\infty}} \lesssim \sup _{j \in \boldsymbol{Z}}\|\|\left(I-\Delta_{\xi}\right)^{s / 2} m_{j}(\xi, \eta)\left\|_{L_{\xi}^{2}}\right\|_{L_{\eta}^{\infty}}, \\
& \sup _{j \in \boldsymbol{Z}}\|\|\left(I-\Delta_{\eta}\right)^{s / 2} \widetilde{m}_{j}(\xi, \eta)\left\|_{L_{\eta}^{2}}\right\|_{L_{\xi}^{\infty}} \lesssim \sup _{j \in \boldsymbol{Z}}\|\|\left(I-\Delta_{\eta}\right)^{s / 2} m_{j}(\xi, \eta)\left\|_{L_{\eta}^{2}}\right\|_{L_{\xi}^{\infty}},
\end{aligned}
$$

where $m_{j}$ is defined by (1.3).

PROOF. We only consider the first inequality, because the second one can be proved in the same way. We may assume that $\operatorname{supp} \widetilde{\Psi} \subset\left\{2^{-k_{0}} \leq\left(|\xi|^{2}+|\eta|^{2}\right)^{1 / 2} \leq 2^{k_{0}}\right\}$ with $k_{0} \in N_{0}$.

We first prove the following inequality:

$$
\begin{aligned}
\|\|(I & \left.-\Delta_{\xi}\right)^{s / 2}(F G)(\xi, \eta)\left\|_{L_{\xi}^{2}}\right\|_{L_{\eta}^{\infty}} \\
& \lesssim\|\|\left(I-\Delta_{\xi}\right)^{s / 2} F(\xi, \eta)\left\|_{L_{\xi}^{2}}\right\|_{L_{\eta}^{\infty}}\|\|\left(1+|x|^{2}\right)^{|s| / 2} \mathcal{F}_{1} G(x, \eta)\left\|_{L_{x}^{1}}\right\|_{L_{\eta}^{\infty}},
\end{aligned}
$$

where $\mathcal{F}_{1} G(x, \eta)$ is the partial Fourier transform of $G(\xi, \eta)$ with respect to the $\xi$-variable. Since

$$
\begin{aligned}
& \left(1+|x|^{2}\right)^{s / 2}\left|\mathcal{F}_{1}(F G)(x, \eta)\right| \\
& \quad \lesssim \int_{\boldsymbol{R}^{n}}\left(1+|x-y|^{2}\right)^{s / 2}\left|\mathcal{F}_{1} F(x-y, \eta)\right|\left(1+|y|^{2}\right)^{|s| / 2}\left|\mathcal{F}_{1} G(y, \eta)\right| d y,
\end{aligned}
$$

it follows from Plancherel's theorem and Young's inequality that

$$
\begin{aligned}
\|(I- & \left.\Delta_{\xi}\right)^{s / 2}(F G)(\xi, \eta)\left\|_{L_{\xi}^{2}}=(2 \pi)^{-n / 2}\right\|\left(1+|x|^{2}\right)^{s / 2} \mathcal{F}_{1}(F G)(x, \eta) \|_{L_{x}^{2}} \\
& \lesssim\left\|\left(1+|x|^{2}\right)^{s / 2} \mathcal{F}_{1} F(x, \eta)\right\|_{L_{x}^{2}}\left\|\left(1+|x|^{2}\right)^{|s| / 2} \mathcal{F}_{1} G(x, \eta)\right\|_{L_{x}^{1}} \\
& =(2 \pi)^{n / 2}\left\|\left(I-\Delta_{\xi}\right)^{s / 2} F(\xi, \eta)\right\|_{L_{\xi}^{2}}\left\|\left(1+|x|^{2}\right)^{|s| / 2} \mathcal{F}_{1} G(x, \eta)\right\|_{L_{x}^{1}}
\end{aligned}
$$

which implies (3.1).

Since supp $\Psi\left(\xi / 2^{k}, \eta / 2^{k}\right) \subset\left\{2^{k-1} \leq\left(|\xi|^{2}+|\eta|^{2}\right)^{1 / 2} \leq 2^{k+1}\right\}$, we have

$$
\begin{aligned}
\tilde{m}_{j}(\xi, \eta) & =m\left(2^{j} \xi, 2^{j} \eta\right) \Phi\left(2^{j} \xi, 2^{j} \eta\right) \widetilde{\Psi}(\xi, \eta) \\
& =\sum_{k=-k_{0}}^{k_{0}} m\left(2^{j} \xi, 2^{j} \eta\right) \Psi\left(\xi / 2^{k}, \eta / 2^{k}\right) \Phi\left(2^{j} \xi, 2^{j} \eta\right) \widetilde{\Psi}(\xi, \eta) \\
& =\sum_{k=-k_{0}}^{k_{0}} m_{j+k}\left(\xi / 2^{k}, \eta / 2^{k}\right) \Phi_{j}(\xi, \eta),
\end{aligned}
$$


where $m_{j+k}$ is defined by (1.3) and $\Phi_{j}(\xi, \eta)=\Phi\left(2^{j} \xi, 2^{j} \eta\right) \widetilde{\Psi}(\xi, \eta)$. Combining this with (3.1), we see that

$$
\begin{aligned}
\|\|\left(I-\Delta_{\xi}\right)^{s / 2} \tilde{m}_{j}(\xi, \eta)\left\|_{L_{\xi}^{2}}\right\|_{L_{\eta}^{\infty}} \lesssim & \sum_{k=-k_{0}}^{k_{0}}\|\|\left(I-\Delta_{\xi}\right)^{s / 2}\left[m_{j+k}\left(\cdot / 2^{k}, \cdot / 2^{k}\right)\right](\xi, \eta)\left\|_{L_{\xi}^{2}}\right\|_{L_{\eta}^{\infty}} \\
& \times\|\|\left(1+|x|^{2}\right)^{|s| / 2} \mathcal{F}_{1} \Phi_{j}(x, \eta)\left\|_{L_{x}^{1}}\right\|_{L_{\eta}^{\infty}} .
\end{aligned}
$$

By a change of variables, for $|k| \leq k_{0}$,

$$
\begin{aligned}
\|\|\left(I-\Delta_{\xi}\right)^{s / 2}\left[m_{j+k}\left(\cdot / 2^{k}, \cdot / 2^{k}\right)\right](\xi, \eta)\left\|_{L_{\xi}^{2}}\right\|_{L_{\eta}^{\infty}} & \lesssim\|\|\left(I-\Delta_{\xi}\right)^{s / 2} m_{j+k}(\xi, \eta)\left\|_{L_{\xi}^{2}}\right\|_{L_{\eta}^{\infty}} \\
& \leq \sup _{j \in \boldsymbol{Z}}\|\|\left(I-\Delta_{\xi}\right)^{s / 2} m_{j}(\xi, \eta)\left\|_{L_{\xi}^{2}}\right\|_{L_{\eta}^{\infty}},
\end{aligned}
$$

where the implicit constant is independent of $j$. On the other hand, since

$$
\left|\partial_{\xi}^{\alpha} \Phi_{j}(\xi, \eta)\right| \lesssim \chi_{\left\{|\xi| \leq 2^{\left.k_{0}\right\}}\right.}(\xi, \eta)
$$

for each $\alpha$, we see that

$$
\sup _{j \in \boldsymbol{Z}}\|\|\left(1+|x|^{2}\right)^{|s| / 2} \mathcal{F}_{1} \Phi_{j}(x, \eta)\left\|_{L_{x}^{1}}\right\|_{L_{\eta}^{\infty}}<\infty .
$$

Therefore, we obtain the desired result.

One of the ideas in our proof of Theorem 1.1 is to decompose $m$ as $m(\xi, \eta)=$ $\sum_{j \in \boldsymbol{Z}} m_{j}\left(\xi / 2^{j}, \eta / 2^{j}\right)$ and use pointwise estimate of $T_{m_{j}\left(\cdot / 2^{j}, \cdot / 2^{j}\right)}(f, g)(x)$. The pointwise estimate is based on the following two lemmas.

LEMMA 3.2. Let $s>n / 2$ and $\max \{1, n / s\}<q<2$. If $\operatorname{supp} \sigma \subset\{(x, \xi) ;|\xi| \leq 10\}$, then

$$
|\sigma(X, D / r) f(x)| \lesssim\left\|\left(I-\Delta_{\xi}\right)^{s / 2} \sigma(x, \xi)\right\|_{L_{\xi}^{2}} M_{q} f(x)
$$

for all $r>0$. The implicit constant is independent of $r$ and $f \in \mathcal{S}\left(\boldsymbol{R}^{n}\right)$.

PROOF. Using the formula

$$
\sigma(X, D / r) f(x)=\int_{\boldsymbol{R}^{n}} r^{n} \mathcal{F}_{2}^{-1} \sigma(x, r(x-y)) f(y) d y,
$$

where $\mathcal{F}_{2}^{-1} \sigma(x, y)$ is the partial inverse Fourier transform of $\sigma(x, \xi)$ with respect to the $\xi$ variable, we have by Hölder's inequality

$$
\begin{aligned}
|\sigma(X, D / r) f(x)| \leq & \left(\int_{\boldsymbol{R}^{n}}\left|(1+r|y|)^{s} \mathcal{F}_{2}^{-1} \sigma(x, r y)\right|^{q^{\prime}} r^{n} d y\right)^{1 / q^{\prime}} \\
& \times\left(\int_{\boldsymbol{R}^{n}}\left|\frac{f(y)}{(1+r|x-y|)^{s}}\right|^{q} r^{n} d y\right)^{1 / q} .
\end{aligned}
$$


Note that $q^{\prime}>2$, and then Lemma 2.2 gives

$$
\begin{aligned}
\left(\int_{\boldsymbol{R}^{n}}\left|(1+r|y|)^{s} \mathcal{F}_{2}^{-1} \sigma(x, r y)\right|^{q^{\prime}} r^{n} d y\right)^{1 / q^{\prime}} & =\left(\int_{\boldsymbol{R}^{n}}\left|(1+|y|)^{s} \mathcal{F}_{2}^{-1} \sigma(x, y)\right|^{q^{\prime}} d y\right)^{1 / q^{\prime}} \\
& \lesssim\left\|\left(I-\Delta_{\xi}\right)^{s / 2} \sigma(x, \xi)\right\|_{L_{\xi}^{2}}
\end{aligned}
$$

Since $s q>n$, we also have

$$
\left(\int_{\boldsymbol{R}^{n}}\left|\frac{f(y)}{(1+r|x-y|)^{s}}\right|^{q} r^{n} d y\right)^{1 / q} \lesssim M\left(|f|^{q}\right)(x)^{1 / q}=M_{q} f(x) .
$$

Combining these inequalities, we obtain the desired result.

LEMmA 3.3. Let $s>n / 2$ and $\max \{1, n / s\}<q<2$. If $\operatorname{supp} m \subset\{(\xi, \eta) ;|\xi| \leq 10\}$, then

$$
\left|T_{m(\cdot / r, \cdot / r)}(f, g)(x)\right| \lesssim M_{q} f(x)\left\|\int_{\boldsymbol{R}^{n}} e^{i x \cdot \eta}\left(I-\Delta_{\xi}\right)^{s / 2} m(\xi, \eta / r) \widehat{g}(\eta) d \eta\right\|_{L_{\xi}^{2}}
$$

for all $r>0$. Similarly, if $\operatorname{supp} m \subset\{(\xi, \eta) ;|\eta| \leq 10\}$, then

$$
\left|T_{m(\cdot / r, \cdot / r)}(f, g)(x)\right| \lesssim M_{q} g(x)\left\|\int_{\boldsymbol{R}^{n}} e^{i x \cdot \xi}\left(I-\Delta_{\eta}\right)^{s / 2} m(\xi / r, \eta) \widehat{f}(\xi) d \xi\right\|_{L_{\eta}^{2}}
$$

for all $r>0$. The implicit constants in the above inequalities are independent of $r$ and $f, g \in \mathcal{S}\left(\boldsymbol{R}^{n}\right)$.

PROOF. By symmetry of the situation, we consider only the case $\operatorname{supp} m \subset$ $\{(\xi, \eta) ;|\xi| \leq 10\}$

We write the bilinear operator $T_{m(\cdot / r, \cdot / r)}$ as a combination of linear operators in the following form:

$$
\begin{aligned}
T_{m(\cdot / r, \cdot r)}(f, g)(x) & =\frac{1}{(2 \pi)^{n}} \int_{\boldsymbol{R}^{n}} e^{i x \cdot \xi}\left(\frac{1}{(2 \pi)^{n}} \int_{\boldsymbol{R}^{n}} e^{i x \cdot \eta} m(\xi / r, \eta / r) \widehat{g}(\eta) d \eta\right) \widehat{f}(\xi) d \xi \\
& =\sigma_{r}(g ; X, D / r) f(x),
\end{aligned}
$$

where

$$
\sigma_{r}(g ; x, \xi)=\frac{1}{(2 \pi)^{n}} \int_{\boldsymbol{R}^{n}} e^{i x \cdot \eta} m(\xi, \eta / r) \widehat{g}(\eta) d \eta .
$$

Since $\operatorname{supp} \sigma_{r}(g ; x, \xi) \subset\{(x, \xi) ;|\xi| \leq 10\}$, Lemma 3.2 gives

$$
\begin{aligned}
\left|T_{m(\cdot / r, \cdot / r)}(f, g)(x)\right|=\left|\sigma_{r}(g ; X, D / r) f(x)\right| \\
\quad \lesssim\left\|\left(I-\Delta_{\xi}\right)^{s / 2} \sigma_{r}(g ; x, \xi)\right\|_{L_{\xi}^{2} M_{q} f(x)} \\
\quad=\left\|\frac{1}{(2 \pi)^{n}} \int_{\boldsymbol{R}^{n}} e^{i x \cdot \eta}\left(I-\Delta_{\xi}\right)^{s / 2} m(\xi, \eta / r) \widehat{g}(\eta) d \eta\right\|_{L_{\xi}^{2}} M_{q} f(x) .
\end{aligned}
$$

The proof is complete. 
Proof OF TheOREM 1.1. We assume that $m(\xi, \eta)$ satisfies

$$
\begin{aligned}
& \sup _{j \in \boldsymbol{Z}}\left(\|\|\left(I-\Delta_{\xi}\right)^{s / 2} m_{j}(\xi, \eta)\left\|_{L_{\xi}^{2}}\right\|_{L_{\eta}^{\infty}}+\|\|\left(I-\Delta_{\eta}\right)^{s / 2} m_{j}(\xi, \eta)\left\|_{L_{\eta}^{2}}\right\|_{L_{\xi}^{\infty}}\right) \\
& \quad=A<\infty,
\end{aligned}
$$

where $s>n / 2$ and $m_{j}$ is defined by (1.3). By Lemma 2.1, we can decompose $m$ as

$$
m=m \Phi_{(1,1)}+m \Phi_{(0,1)}+m \Phi_{(1,0)}=m^{(1)}+m^{(2)}+m^{(3)} .
$$

Note that

$$
\begin{aligned}
& \operatorname{supp} m^{(1)} \subset\left\{(\xi, \eta) \in \boldsymbol{R}^{n} \times \boldsymbol{R}^{n} ;|\xi| / 8 \leq|\eta| \leq 8|\xi|\right\}, \\
& \operatorname{supp} m^{(2)} \subset\left\{(\xi, \eta) \in \boldsymbol{R}^{n} \times \boldsymbol{R}^{n} ;|\xi| \leq|\eta| / 2\right\}, \\
& \operatorname{supp} m^{(3)} \subset\left\{(\xi, \eta) \in \boldsymbol{R}^{n} \times \boldsymbol{R}^{n} ;|\eta| \leq|\xi| / 2\right\} .
\end{aligned}
$$

By Lemma 3.1, we have

$$
\sup _{j \in \boldsymbol{Z}}\left(\|\|\left(I-\Delta_{\xi}\right)^{s / 2} m_{j}^{(i)}(\xi, \eta)\left\|_{L_{\xi}^{2}}\right\|_{L_{\eta}^{\infty}}+\|\|\left(I-\Delta_{\eta}\right)^{s / 2} m_{j}^{(i)}(\xi, \eta)\left\|_{L_{\eta}^{2}}\right\|_{L_{\xi}^{\infty}}\right) \lesssim A
$$

for $i=1,2,3$, where $m_{j}^{(i)}(\xi, \eta)=m^{(i)}\left(2^{j} \xi, 2^{j} \eta\right) \Psi(\xi, \eta)$. It is sufficient to prove the estimate $\left\|T_{m^{(i)}}\right\|_{L^{2} \times L^{2} \rightarrow L^{1}} \lesssim A$ for $i=1,2,3$. By symmetry of the situation, $m^{(2)}$ and $m^{(3)}$ are treated in the same way. Thus, we shall only consider $m^{(1)}$ and $m^{(2)}$.

Since $n / s<2$, we can take $q$ satisfying $\max \{1, n / s\}<q<2$. We denote by $\mathcal{A}$ the set of $\psi \in \mathcal{S}\left(\boldsymbol{R}^{n}\right)$ for which supp $\psi$ is a compact subset of $\boldsymbol{R}^{n} \backslash\{0\}$. Since

Estimate for $T_{m^{(1)}}$ : We simply write $m$ and $m_{j}$ instead of $m^{(1)}$ and $m_{j}^{(1)}$, respectively.

$$
m(\xi, \eta)=\sum_{j \in \boldsymbol{Z}} m(\xi, \eta) \Psi\left(\xi / 2^{j}, \eta / 2^{j}\right)=\sum_{j=-\infty}^{\infty} m_{j}\left(\xi / 2^{j}, \eta / 2^{j}\right),
$$

we have

$$
T_{m}=\sum_{j \in \boldsymbol{Z}} T_{m_{j}\left(\cdot / 2^{j}, \cdot / 2^{j}\right)} .
$$

In the present case $\left(m=m^{(1)}\right)$, an important fact is that $|\xi| \approx|\eta|$ for $(\xi, \eta) \in \operatorname{supp} m$. This implies that

$$
m_{j}\left(\xi / 2^{j}, \eta / 2^{j}\right) \neq 0 \Rightarrow|\xi| \approx|\eta| \approx 2^{j} .
$$

By this fact, we can take a $\psi \in \mathcal{A}$ such that $\psi\left(\xi / 2^{j}\right)=\psi\left(\eta / 2^{j}\right)=1$ for all $(\xi, \eta)$ satisfying $m_{j}\left(\xi / 2^{j}, \eta / 2^{j}\right) \neq 0$. Thus $T_{m_{j}\left(\cdot / 2^{j}, \cdot 2^{j}\right)}(f, g)(x)$ can be written as

$$
\begin{aligned}
& T_{m_{j}\left(\cdot / 2^{j}, \cdot / 2^{j}\right)}(f, g)(x) \\
& \quad=\frac{1}{(2 \pi)^{2 n}} \int_{\boldsymbol{R}^{2 n}} e^{i x \cdot(\xi+\eta)} m_{j}\left(\xi / 2^{j}, \eta / 2^{j}\right) \psi\left(\xi / 2^{j}\right) \widehat{f}(\xi) \psi\left(\eta / 2^{j}\right) \widehat{g}(\eta) d \xi d \eta \\
& \quad=T_{m_{j}\left(\cdot / 2^{j}, \cdot / 2^{j}\right)}\left(f_{j}, g_{j}\right)(x),
\end{aligned}
$$

where $f_{j}=\psi\left(D / 2^{j}\right) f$ and $g_{j}=\psi\left(D / 2^{j}\right) g$. 
We shall estimate the $L^{1}$-norm of $T_{m_{j}\left(\cdot / 2^{j}, \cdot / 2^{j}\right)}\left(f_{j}, g_{j}\right)$. Note that $\operatorname{supp} m_{j} \subset$ $\left\{(\xi, \eta) ;|\xi|^{2}+|\eta|^{2} \leq 4\right\}$ for all $j \in \boldsymbol{Z}$. Hence, by Lemma 3.3,

$$
\left|T_{m_{j}\left(\cdot / 2^{j}, \cdot / 2^{j}\right)}\left(f_{j}, g_{j}\right)(x)\right| \lesssim M_{q}\left(f_{j}\right)(x)\left\|\int_{\boldsymbol{R}^{n}} e^{i x \cdot \eta}\left(I-\Delta_{\xi}\right)^{s / 2} m_{j}\left(\xi, \eta / 2^{j}\right) \widehat{g}_{j}(\eta) d \eta\right\|_{L_{\xi}^{2}} .
$$

Integrating with respect to $x$ and using Schwarz's inequality, we obtain

$$
\left\|T_{m_{j}\left(\cdot / 2^{j}, \cdot / 2^{j}\right)}\left(f_{j}, g_{j}\right)\right\|_{L^{1}} \lesssim\left\|M_{q}\left(f_{j}\right)\right\|_{L^{2}}\left\|\int_{\boldsymbol{R}^{n}} e^{i x \cdot \eta}\left(I-\Delta_{\xi}\right)^{s / 2} m_{j}\left(\xi, \eta / 2^{j}\right) \widehat{g}_{j}(\eta) d \eta\right\|_{L_{\xi, x}^{2}} .
$$

For the former $L^{2}$-norm, we have $\left\|M_{q}\left(f_{j}\right)\right\|_{L^{2}} \lesssim\left\|f_{j}\right\|_{L^{2}}$ since $q<2$. For the latter $L^{2}$-norm, Plancherel's theorem and (3.3) give

$$
\begin{gathered}
\left\|\int_{\boldsymbol{R}^{n}} e^{i x \cdot \eta}\left(I-\Delta_{\xi}\right)^{s / 2} m_{j}\left(\xi, \eta / 2^{j}\right) \widehat{g}_{j}(\eta) d \eta\right\|_{L_{\xi, x}^{2}} \\
=(2 \pi)^{n / 2}\left\|\left(I-\Delta_{\xi}\right)^{s / 2} m_{j}\left(\xi, \eta / 2^{j}\right) \widehat{g}_{j}(\eta)\right\|_{L_{\eta, \xi}^{2}} \\
\lesssim A\left\|\widehat{g}_{j}(\eta)\right\|_{L_{\eta}^{2}}=(2 \pi)^{n / 2} A\left\|g_{j}\right\|_{L^{2}} .
\end{gathered}
$$

Thus

$$
\left\|T_{m_{j}\left(\cdot / 2^{j}, \cdot / 2^{j}\right)}\left(f_{j}, g_{j}\right)\right\|_{L^{1}} \lesssim A\left\|f_{j}\right\|_{L^{2}}\left\|g_{j}\right\|_{L^{2}}
$$

Hence, using Schwarz's inequality, we obtain

$$
\begin{aligned}
& \left\|T_{m}(f, g)\right\|_{L^{1}}=\left\|\sum_{j \in \boldsymbol{Z}} T_{m_{j}\left(\cdot / 2^{j}, \cdot / 2^{j}\right)}\left(f_{j}, g_{j}\right)\right\|_{L^{1}} \leq \sum_{j \in \boldsymbol{Z}}\left\|T_{m_{j}\left(\cdot / 2^{j}, \cdot / 2^{j}\right)}\left(f_{j}, g_{j}\right)\right\|_{L^{1}} \\
& \quad \lesssim A \sum_{j \in \boldsymbol{Z}}\left\|f_{j}\right\|_{L^{2}}\left\|g_{j}\right\|_{L^{2}} \leq A\left(\sum_{j \in \boldsymbol{Z}}\left\|f_{j}\right\|_{L^{2}}^{2}\right)^{1 / 2}\left(\sum_{j \in \boldsymbol{Z}}\left\|g_{j}\right\|_{L^{2}}^{2}\right)^{1 / 2} \\
& \quad \lesssim A\|f\|_{L^{2}}\|g\|_{L^{2}},
\end{aligned}
$$

where the last inequality holds because $\psi$ has compact support away from the origin. This proves the boundedness of $T_{m^{(1)}}$.

Estimate for $T_{m^{(2)}}$ : We simply write $m$ and $m_{j}$ instead of $m^{(2)}$ and $m_{j}^{(2)}$, respectively. In the present case $\left(m=m^{(2)}\right)$, an important fact is that $|\xi| \lesssim|\eta| \approx|\xi+\eta|$ for $(\xi, \eta) \in \operatorname{supp} m$. This implies that

$$
m_{j}\left(\xi / 2^{j}, \eta / 2^{j}\right) \neq 0 \Rightarrow|\eta| \approx|\xi+\eta| \approx 2^{j}
$$

By (3.5), we can take a $\psi \in \mathcal{A}$ such that $\psi\left(\eta / 2^{j}\right)=1$ for all $(\xi, \eta)$ satisfying $m_{j}\left(\xi / 2^{j}, \eta / 2^{j}\right) \neq$ 0 . Thus $T_{m_{j}\left(\cdot / 2^{j}, \cdot / 2^{j}\right)}(f, g)(x)$ can be written as

$$
\begin{aligned}
& T_{m_{j}\left(\cdot / 2^{j}, \cdot / 2^{j}\right)}(f, g)(x) \\
& \quad=\frac{1}{(2 \pi)^{2 n}} \int_{\boldsymbol{R}^{2 n}} e^{i x \cdot(\xi+\eta)} m_{j}\left(\xi / 2^{j}, \eta / 2^{j}\right) \widehat{f}(\xi) \psi\left(\eta / 2^{j}\right) \widehat{g}(\eta) d \xi d \eta \\
& \quad=T_{m_{j}\left(\cdot / 2^{j}, \cdot / 2^{j}\right)}\left(f, g_{j}\right)(x),
\end{aligned}
$$


where $g_{j}=\psi\left(D / 2^{j}\right) g$. From (3.5), it also follows that there exists a constant $B>1$ such that the Fourier transform of $T_{m_{j}\left(\cdot / 2^{j}, \cdot / 2^{j}\right)}(f, g)(x)$ is included in the annulus $\left\{\zeta \in \boldsymbol{R}^{n} ; B^{-1} 2^{j} \leq\right.$ $\left.|\zeta| \leq B 2^{j}\right\}$

Using the above facts and using Lemma 2.4, we can estimate the $L^{1}$-norm of $T_{m}(f, g)$ as follows:

$$
\begin{aligned}
\left\|T_{m}(f, g)\right\|_{L^{1}} & \lesssim\left\|T_{m}(f, g)\right\|_{H^{1}}=\left\|\sum_{j \in \boldsymbol{Z}} T_{m_{j}\left(\cdot / 2^{j}, \cdot / 2^{j}\right)}\left(f, g_{j}\right)\right\|_{H^{1}} \\
& \lesssim\left\|\left(\sum_{j \in \boldsymbol{Z}}\left|T_{m_{j}\left(\cdot / 2^{j}, \cdot / 2^{j}\right)}\left(f, g_{j}\right)\right|^{2}\right)^{1 / 2}\right\|_{L^{1}} \cdot
\end{aligned}
$$

Since $\operatorname{supp} m_{j} \subset\left\{(\xi, \eta) ;|\xi|^{2}+|\eta|^{2} \leq 4\right\}$ for all $j \in Z$, Lemma 3.3 yields

$$
\left|T_{m_{j}\left(\cdot / 2^{j}, \cdot / 2^{j}\right)}\left(f, g_{j}\right)(x)\right| \lesssim M_{q} f(x)\left\|\int_{\boldsymbol{R}^{n}} e^{i x \cdot \eta}\left(I-\Delta_{\xi}\right)^{s / 2} m_{j}\left(\xi, \eta / 2^{j}\right) \widehat{g}_{j}(\eta) d \eta\right\|_{L_{\xi}^{2}} .
$$

Taking $\ell^{2}$-norm with respect to $j \in Z$, we have

$$
\begin{aligned}
& \left(\sum_{j \in \boldsymbol{Z}}\left|T_{m_{j}\left(\cdot / 2^{j}, \cdot / 2^{j}\right)}\left(f, g_{j}\right)(x)\right|^{2}\right)^{1 / 2} \\
& \quad \lesssim M_{q} f(x)\left(\sum_{j \in \boldsymbol{Z}}\left\|\int_{\boldsymbol{R}^{n}} e^{i x \cdot \eta}(I-\Delta \xi)^{s / 2} m_{j}\left(\xi, \eta / 2^{j}\right) \widehat{g}_{j}(\eta) d \eta\right\|_{L_{\xi}^{2}}\right)^{1 / 2} .
\end{aligned}
$$

Thus, by Schwarz's inequality,

$$
\begin{aligned}
& \left\|\left(\sum_{j \in \boldsymbol{Z}}\left|T_{m_{j}\left(\cdot / 2^{j}, \cdot / 2^{j}\right)}\left(f, g_{j}\right)(x)\right|^{2}\right)^{1 / 2}\right\|_{L^{1}} \\
& \quad \lesssim\left\|M_{q} f\right\|_{L^{2}}\left(\sum_{j \in \boldsymbol{Z}}\left\|\int_{\boldsymbol{R}^{n}} e^{i x \cdot \eta}\left(I-\Delta_{\xi}\right)^{s / 2} m_{j}\left(\xi, \eta / 2^{j}\right) \widehat{g_{j}}(\eta) d \eta\right\|_{L_{\xi, x}^{2}}^{2}\right)^{1 / 2} .
\end{aligned}
$$

For the former $L^{2}$-norm, we have $\left\|M_{q} f\right\|_{L^{2}} \lesssim\|f\|_{L^{2}}$ since $q<2$. For the latter $L^{2}$-norm, Plancherel's theorem and (3.3) give

$$
\begin{aligned}
\sum_{j \in \boldsymbol{Z}} \| & \int_{\boldsymbol{R}^{n}} e^{i x \cdot \eta}\left(I-\Delta_{\xi}\right)^{s / 2} m_{j}\left(\xi, \eta / 2^{j}\right) \widehat{g}_{j}(\eta) d \eta \|_{L_{\xi, x}^{2}}^{2} \\
& =(2 \pi)^{n} \sum_{j \in \boldsymbol{Z}}\left\|\left(I-\Delta_{\xi}\right)^{s / 2} m_{j}\left(\xi, \eta / 2^{j}\right) \widehat{g}_{j}(\eta)\right\|_{L_{\eta, \xi}^{2}}^{2} \\
& \lesssim A^{2} \sum_{j \in \boldsymbol{Z}}\left\|\widehat{g}_{j}(\eta)\right\|_{L_{\eta}^{2}}^{2}=(2 \pi)^{n} A^{2} \sum_{j \in \boldsymbol{Z}}\left\|g_{j}\right\|_{L^{2}}^{2} \lesssim A^{2}\|g\|_{L^{2}}^{2},
\end{aligned}
$$

where the last inequality holds because $\psi$ has compact support away from the origin. Combining the above inequalities, we obtain the desired estimate. This completes the proof of Theorem 1.1. 
REMARK 3.4. For the multiplier $m^{(2)}$ appearing in the proof of Theorem 1.1, we have actually proved

$$
\left\|T_{m^{(2)}}\right\|_{L^{2} \times L^{2} \rightarrow H^{1}} \lesssim A
$$

where $A$ is the same as in (3.2). By symmetry of the situation, $T_{m^{(3)}}$ has the same boundedness property.

4. Proof of Theorem 1.2. The former part of Theorem 1.2 readily follows from Theorem 1.1 and Theorem A by duality and interpolation. To see this, we use the following lemma.

LEMMA 4.1. Let $s>0$ and $\varepsilon>0$. If $\operatorname{supp} F \subset\left\{\left(|\xi|^{2}+|\eta|^{2}\right)^{1 / 2} \leq 10\right\}$, then

$$
\|\|\left(I-\Delta_{\xi}\right)^{s / 2} F(\xi, \eta)\left\|_{L_{\xi}^{2}}\right\|_{L_{\eta}^{\infty}}+\|\|\left(I-\Delta_{\eta}\right)^{s / 2} F(\xi, \eta)\left\|_{L_{\eta}^{2}}\right\|_{L_{\xi}^{\infty}} \lesssim\|F(\xi, \eta)\|_{B_{\infty, \infty}^{s+\varepsilon}} .
$$

Proof. Let $\varphi \in \mathcal{S}\left(\boldsymbol{R}^{n}\right)$ be such that $\varphi=1$ on $\left\{\xi \in \boldsymbol{R}^{n} ;|\xi| \leq 10\right\}$. Since $s>0$ and $F(\xi, \eta)=\varphi(\xi) F(\xi, \eta)$, it follows from Lemma 2.3 that

$$
\begin{aligned}
\left\|\left(I-\Delta_{\xi}\right)^{s / 2} F(\xi, \eta)\right\|_{L^{2}\left(\boldsymbol{R}_{\xi}^{n}\right)} & \approx\|F(\xi, \eta)\|_{B_{2,2}^{s}\left(\boldsymbol{R}_{\xi}^{n}\right)}=\|\varphi(\xi) F(\xi, \eta)\|_{B_{2,2}^{s}\left(\boldsymbol{R}_{\xi}^{n}\right)} \\
& \lesssim\|\varphi(\xi)\|_{B_{2,2}^{s}\left(\boldsymbol{R}_{\xi}^{n}\right)}\|F(\xi, \eta)\|_{B_{\infty, 2}^{s}\left(\boldsymbol{R}_{\xi}^{n}\right)}
\end{aligned}
$$

We denote by $\left\{\psi_{j}\right\}_{j=0}^{\infty}$ and $\left\{\Psi_{j}\right\}_{j=0}^{\infty}$ the sequences of functions used in the definition of Besov spaces on $\boldsymbol{R}^{n}$ and $\boldsymbol{R}^{2 n}$, respectively. Then

$$
\begin{aligned}
\|F(\xi, \eta)\|_{B_{\infty, 2}^{s}\left(\boldsymbol{R}_{\xi}^{n}\right)} & \leq\|F(\xi, \eta)\|_{B_{\infty, 1}^{s}\left(\boldsymbol{R}_{\xi}^{n}\right)} \\
& =\sum_{j=0}^{\infty} 2^{j s}\left\|\psi_{j}\left(D_{\xi}\right) F(\xi, \eta)\right\|_{L^{\infty}\left(\boldsymbol{R}_{\xi}^{n}\right)} \\
& \leq \sum_{k=0}^{\infty} \sum_{j=0}^{\infty} 2^{j s}\left\|\psi_{j}\left(D_{\xi}\right) \Psi_{k}\left(D_{\xi, \eta}\right) F(\xi, \eta)\right\|_{L^{\infty}\left(\boldsymbol{R}_{\xi}^{n}\right)} .
\end{aligned}
$$

Since $\psi_{j}(\xi) \Psi_{k}(\xi, \eta)=0$ if $j \geq k+2$, the last sum can be estimated as

$$
\begin{aligned}
& \sum_{k=0}^{\infty} \sum_{j=0}^{k+1} 2^{j s}\left\|\psi_{j}\left(D_{\xi}\right) \Psi_{k}\left(D_{\xi, \eta}\right) F(\xi, \eta)\right\|_{L^{\infty}\left(\boldsymbol{R}_{\xi}^{n}\right)} \\
& \quad \leq \sum_{k=0}^{\infty} \sum_{j=0}^{k+1} 2^{j s}\left\|\mathcal{F}^{-1} \psi_{j}\right\|_{L^{1}}\left\|\Psi_{k}\left(D_{\xi, \eta}\right) F(\xi, \eta)\right\|_{L^{\infty}\left(\boldsymbol{R}_{\xi}^{n}\right)} \\
& \quad \lesssim \sum_{k=0}^{\infty} 2^{k s}\left\|\Psi_{k}\left(D_{\xi, \eta}\right) F(\xi, \eta)\right\|_{L^{\infty}\left(\boldsymbol{R}_{\xi}^{n}\right)} \\
& \quad \lesssim \sup _{k \in Z} 2^{k(s+\varepsilon)}\left\|\Psi_{k}\left(D_{\xi, \eta}\right) F(\xi, \eta)\right\|_{L^{\infty}\left(\boldsymbol{R}_{\xi, \eta}^{n}\right)}=\|F\|_{B_{\infty, \infty}^{s+\varepsilon}}
\end{aligned}
$$

Combining these inequalities, we obtain the desired result for the term \|\|$\cdot\left\|_{L_{\xi}^{2}}\right\|_{L_{\eta}^{\infty}}$. Interchanging the roles of $\xi$ and $\eta$, we can estimate the term \|\|$\cdot\left\|_{L_{\eta}^{2}}\right\|_{L_{\xi}^{\infty}}$ in the same way. 
PROOF OF THE FORMER PART OF THEOREM 1.2. We shall divide the proof into two steps.

Step 1. Here we give a general result for interpolation. We write $\zeta=(\xi, \eta) \in \boldsymbol{R}^{n} \times \boldsymbol{R}^{n}$. Let $X_{v}, v=0,1$, be Banach spaces satisfying $\mathcal{S}\left(\boldsymbol{R}^{2 n}\right) \hookrightarrow X_{v} \hookrightarrow \mathcal{S}^{\prime}\left(\boldsymbol{R}^{2 n}\right)$, and let

$$
X_{\theta}=\left(X_{0}, X_{1}\right)_{\theta}, \quad 0<\theta<1,
$$

be the complex interpolation spaces of $X_{0}$ and $X_{1}$ in the sense of Triebel [20, 2.4.4]. We assume that $X_{v}, v=0,1$, have the following properties:

$$
\|F(\zeta) \Theta(\zeta)\|_{X_{v}} \lesssim\|F(\zeta)\|_{X_{v}} \quad \text { for each } \Theta \in \mathcal{S}\left(\boldsymbol{R}^{2 n}\right)
$$

where the implicit constant is allowed to depend on $\Theta$, and

$$
\|F(t \zeta)\|_{X_{v}} \lesssim\|F(\zeta)\|_{X_{v}} \text { for all } 1 / 4 \leq t \leq 4
$$

We shall prove that if

$$
\left\|T_{m}\right\|_{H^{p_{v}} \times H^{q_{v}} \rightarrow L^{r_{v}}} \lesssim \sup _{j \in \boldsymbol{Z}}\left\|m_{j}\right\|_{X_{v}}, \quad v=0,1,
$$

then

$$
\left\|T_{m}\right\|_{H^{p_{\theta}} \times H^{q_{\theta}} \rightarrow L^{r}} \lesssim \sup _{j \in \boldsymbol{Z}}\left\|m_{j}\right\|_{X_{\theta}},
$$

where $1 / p_{\theta}=(1-\theta) / p_{0}+\theta / p_{1}, 1 / q_{\theta}=(1-\theta) / q_{0}+\theta / q_{1}$ and $1 / r_{\theta}=(1-\theta) / r_{0}+\theta / r_{1}$.

For $m(\zeta) \in \mathcal{S}\left(\boldsymbol{R}^{2 n}\right)$ and $0<\theta<1$, we set

$$
A_{j}=\left\|m_{j}\right\|_{X_{\theta}}, \quad j \in Z,
$$

where $m_{j}$ is defined by (1.3). By (4.1) and the definition of complex interpolation, there exists an $\mathcal{S}^{\prime}\left(\boldsymbol{R}^{2 n}\right)$-valued function $m_{j, z}$ which is analytic on the strip $S=\{z \in \boldsymbol{C} ; 0<$ $\operatorname{Re} z<1\}$, bounded and continuous on $\bar{S}$ (for precise meanings of analyticity, boundedness, and continuity, see $[20,2.4 .4])$ such that $m_{j, \theta}=m_{j}$ and

$$
\left(\sup _{y \in \boldsymbol{R}}\left\|m_{j, i y}\right\|_{X_{0}}\right)^{1-\theta}\left(\sup _{y \in \boldsymbol{R}}\left\|m_{j, 1+i y}\right\|_{X_{1}}\right)^{\theta} \approx A_{j}
$$

([20, Definition 2.4.4/2, Lemma 2.4.6/3]). Setting

$$
e^{\alpha_{j}}=\frac{\sup _{y \in \boldsymbol{R}}\left\|m_{j, i y}\right\|_{X_{0}}}{\sup _{y \in \boldsymbol{R}}\left\|m_{j, 1+i y}\right\|_{X_{1}}} \quad \text { and } \quad \tilde{m}_{j, z}=e^{\alpha_{j}(z-\theta)} m_{j, z},
$$

we have $\tilde{m}_{j, \theta}=m_{j, \theta}=m_{j}$ and

$$
\sup _{y \in \boldsymbol{R}}\left\|\widetilde{m}_{j, i y}\right\|_{X_{0}}=\sup _{y \in \boldsymbol{R}}\left\|\tilde{m}_{j, 1+i y}\right\|_{X_{1}} \approx A_{j} .
$$

Let $\widetilde{\Psi} \in \mathcal{S}\left(\boldsymbol{R}^{2 n}\right)$ be such that $\operatorname{supp} \widetilde{\Psi} \subset\left\{2^{-2} \leq|\zeta| \leq 2^{2}\right\}$ and $\widetilde{\Psi}=1$ on $\operatorname{supp} \Psi$, where $\Psi$ is the function appearing in (1.3). We define a family of bilinear Fourier multipliers $m_{z}$ by

$$
m_{z}(\zeta)=\sum_{j \in \boldsymbol{Z}} \tilde{m}_{j, z}\left(2^{-j} \zeta\right) \widetilde{\Psi}\left(2^{-j} \zeta\right), \quad z \in \bar{S}
$$


Then $m_{\theta}(\zeta)=\sum_{j \in \mathbf{Z}} m_{j}\left(2^{-j} \zeta\right) \widetilde{\Psi}\left(2^{-j} \zeta\right)=m(\zeta)$. From the support properties of $\Psi$ and $\widetilde{\Psi}$, it follows that

$$
\left(m_{z}\right)_{k}(\zeta)=m_{z}\left(2^{k} \zeta\right) \Psi(\zeta)=\sum_{j=k-2}^{k+2} \tilde{m}_{j, z}\left(2^{-j+k} \zeta\right) \widetilde{\Psi}\left(2^{-j+k} \zeta\right) \Psi(\zeta)
$$

for each $k \in \boldsymbol{Z}$, and consequently

$$
\left\|\left(m_{v+i y}\right)_{k}\right\|_{X_{v}} \leq \sum_{j=k-2}^{k+2}\left\|\tilde{m}_{j, v+i y}\left(2^{-j+k} \zeta\right) \widetilde{\Psi}\left(2^{-j+k} \zeta\right) \Psi(\zeta)\right\|_{X_{v}}
$$

for $v=0,1$. Hence, by (4.2), (4.3) and (4.6),

$$
\left\|\left(m_{v+i y}\right)_{k}\right\|_{X_{v}} \lesssim \sum_{j=k-2}^{k+2}\left\|\tilde{m}_{j, v+i y}\left(2^{-j+k} \cdot\right)\right\|_{X_{v}} \lesssim \sum_{j=k-2}^{k+2}\left\|\tilde{m}_{j, v+i y}\right\|_{X_{v}} \lesssim \sup _{j \in \boldsymbol{Z}} A_{j} .
$$

Combining this with (4.4), we have

$$
\left\|T_{m_{v+i y}}\right\|_{H^{p^{v}} \times H^{q_{\nu}} \rightarrow L^{r_{v}}} \lesssim \sup _{k \in \boldsymbol{Z}}\left\|\left(m_{v+i y}\right)_{k}\right\|_{X_{v}} \lesssim \sup _{j \in \boldsymbol{Z}} A_{j}
$$

Therefore, the interpolation theorem for analytic families of operators ([10, 18]) gives (4.5).

Step 2. We shall introduce a notation. For $s_{0} \in(0, \infty)$ and $t, p, q \in[1, \infty]$, we simply write $\left[s_{0}, 1 / t ; 1 / p, 1 / q\right]$ to mean that the estimate

$$
\left\|T_{m}\right\|_{H^{p} \times H^{q} \rightarrow L^{r}} \lesssim \sup _{j \in \boldsymbol{Z}}\left\|m_{j}\right\|_{B_{t, \infty}^{s}}, \quad 1 / r=1 / p+1 / q,
$$

holds for each $s>s_{0}$ with the implicit constant depending on $s$, where we replace $H^{\infty} \times$ $H^{\infty} \rightarrow L^{\infty}$ by $L^{\infty} \times L^{\infty} \rightarrow B M O$ in the case $p=q=r=\infty$.

We shall apply the general result of Step 1 to the case of the Besov spaces. These spaces have the properties (4.2) and (4.3) (see Lemma 2.3 and [16, Proposition 2.1.3/3], respectively). Also, it is known that

$$
B_{t, \infty}^{s}=\left(B_{t_{0}, \infty}^{s_{0}}, B_{t_{1}, \infty}^{s_{1}}\right)_{\theta},
$$

where $t, t_{0}, t_{1} \in[1, \infty], s, s_{0}, s_{1} \in(0, \infty), 0<\theta<1$, and

$$
1 / t=(1-\theta) / t_{0}+\theta / t_{1}, \quad s=(1-\theta) s_{0}+\theta s_{1}
$$

(see [20, Theorem 2.4.7]). Hence, the interpolation argument in Step 1 implies the following: if $s_{v} \in(0, \infty), t_{v}, p_{v}, q_{v} \in[1, \infty]$, and $\left[s_{v}, 1 / t_{v} ; 1 / p_{v}, 1 / q_{v}\right]$ for $v=0,1$, then $[s, 1 / t ; 1 / p, 1 / q]$ for $s, t, p, q$ given by

$$
(s, 1 / t, 1 / p, 1 / q)=(1-\theta)\left(s_{0}, 1 / t_{0}, 1 / p_{0}, 1 / q_{0}\right)+\theta\left(s_{1}, 1 / t_{1}, 1 / p_{1}, 1 / q_{1}\right)
$$

with $0<\theta<1$.

We also use the duality. Recall the formula

$$
\int_{\boldsymbol{R}^{n}} T_{m}(f, g) h d x=\int_{\boldsymbol{R}^{n}} T_{m^{* 1}}(h, g) f d x=\int_{\boldsymbol{R}^{n}} T_{m^{* 2}}(f, h) g d x,
$$


where $m^{* 1}(\xi, \eta)=m(-(\xi+\eta), \eta)$ and $m^{* 2}(\xi, \eta)=m(\xi,-(\xi+\eta))$ (see [8, p.1155]). This formula says that

$$
\left\|T_{m}\right\|_{L^{p} \times L^{q} \rightarrow L^{r}}=\left\|T_{m^{* 1}}\right\|_{L^{r^{\prime} \times L^{q} \rightarrow L^{p^{\prime}}}}=\left\|T_{m^{* 2}}\right\|_{L^{p} \times L^{r^{\prime} \rightarrow L^{q^{\prime}}}}
$$

for $p, q, r \in[1, \infty]$. Similar equality holds if we replace $L^{1}$ or $L^{\infty}$ by $H^{1}$ or $B M O$ in an appropriate way. Since the Besov space $B_{t, \infty}^{s}$ is invariant under the maps $m \mapsto m^{* i}, i=1,2$, ([16, Proposition 2.1.3/6]), we have the following: if $s \in(0, \infty), t, p, q \in[1, \infty], 1 / r=$ $1 / p+1 / q \leq 1$, and $[s, 1 / t ; 1 / p, 1 / q]$, then $[s, 1 / t ; 1-1 / r, 1 / q]$ and $[s, 1 / t ; 1 / p, 1-1 / r]$.

Now we shall see that the former part of Theorem 1.2 follows from Theorem A and Theorem 1.1. First, Theorem 1.1 and Lemma 4.1 yield $[n / 2,0 ; 1 / 2,1 / 2]$. Then duality gives $[n / 2,0 ; 1 / 2,0]$ and $[n / 2,0 ; 0,1 / 2]$. Hence interpolation between these three points gives $[n / 2,0 ; 1 / p, 1 / q]$ for all $(1 / p, 1 / q) \in J_{0}$. Next, by Theorem A and the embeddings

$$
B_{2, \infty}^{s_{1}+s_{2}+\varepsilon} \hookrightarrow B_{2,2}^{s_{1}+s_{2}}=W^{s_{1}+s_{2}} \hookrightarrow W^{\left(s_{1}, s_{2}\right)},
$$

we have $[n, 1 / 2 ; 1 / p, 1 / q]$ for $(1 / p, 1 / q) \in \partial I_{0}$, where $\partial I_{0}$ denotes the boundary of the region $I_{0}$, which consists of five line segments. Finally, the results for $(1 / p, 1 / q) \in \bigcup_{i=1}^{4} J_{i}$ follow from the results for $(1 / p, 1 / q) \in J_{0}$ and $(1 / p, 1 / q) \in \partial I_{0}$ by interpolation. This completes the proof of the former part of Theorem 1.2.

To prove the latter part of Theorem 1.2, we use the following theorem of [14].

TheOREM B ([14]). Let $s \in \boldsymbol{R}, 1 \leq p, q \leq \infty, 2 / 3 \leq r \leq \infty$, and $1 / p+1 / q=1 / r$. If there exists a positive integer $N$ such that

$$
\left\|T_{m}\right\|_{H^{p}\left(\boldsymbol{R}^{n}\right) \times H^{q}\left(\boldsymbol{R}^{n}\right) \rightarrow L^{r}\left(\boldsymbol{R}^{n}\right)} \lesssim \max _{|\alpha|+|\beta| \leq N}\left(\sup _{\xi, \eta \in \boldsymbol{R}^{n}}(1+|\xi|+|\eta|)^{s}\left|\partial_{\xi}^{\alpha} \partial_{\eta}^{\beta} m(\xi, \eta)\right|\right)
$$

for all $m(\xi, \eta) \in C^{\infty}\left(\boldsymbol{R}^{n} \times \boldsymbol{R}^{n}\right)$, then $s \geq s(p, q)$ with $s(p, q)$ given in Theorem 1.2. In the case $p=q=r=\infty$, the same conclusion holds if we replace $H^{\infty} \times H^{\infty} \rightarrow L^{\infty}$ by $L^{\infty} \times L^{\infty} \rightarrow$ BMO

It should be remarked that in [14] the claim of Theorem B is given for bilinear pseudodifferential operators. But, from its proof, we see that the claim actually holds for bilinear Fourier multiplier operators (see the proofs of [14, Lemma 6.3, Theorem 6.4]).

The following lemma reduces the latter part of Theorem 1.2 to Theorem B.

LEMMA 4.2. Let $s>0$. Then

$$
\sup _{j \in \boldsymbol{Z}}\left\|m_{j}\right\|_{B_{\infty, \infty}^{s}\left(\boldsymbol{R}^{2 n}\right)} \lesssim \max _{|\alpha|+|\beta| \leq[s]+1}\left(\sup _{\xi, \eta \in \boldsymbol{R}^{n}}(1+|\xi|+|\eta|)^{s}\left|\partial_{\xi}^{\alpha} \partial_{\eta}^{\beta} m(\xi, \eta)\right|\right)
$$

for all $m(\xi, \eta) \in C^{\infty}\left(\boldsymbol{R}^{n} \times \boldsymbol{R}^{n}\right)$, where $m_{j}$ is defined by (1.3) and $[s]$ is the integer part of $s$.

Proof. Let $m \in C^{\infty}\left(\boldsymbol{R}^{n} \times \boldsymbol{R}^{n}\right), N=[s]+1$, and

$$
A=\max _{|\gamma| \leq N}\left(\sup _{\zeta \in \boldsymbol{R}^{2 n}}(1+|\zeta|)^{s}\left|\partial^{\gamma} m(\zeta)\right|\right)
$$


Take $0<\theta<1$ such that $s=\theta N$. Then

$$
\begin{aligned}
\left\|m_{j}\right\|_{B_{\infty}^{s}, \infty} & =\sup _{k \in N_{0}}\left(\left\|\Psi_{k}(D) m_{j}\right\|_{L^{\infty}}\right)^{1-\theta}\left(2^{k N}\left\|\Psi_{k}(D) m_{j}\right\|_{L^{\infty}}\right)^{\theta} \\
& \leq\left\|m_{j}\right\|_{B_{\infty, \infty}^{0}}^{1-\theta}\left\|m_{j}\right\|_{B_{\infty, \infty}^{N}}^{\theta} .
\end{aligned}
$$

By the lifting property of Besov spaces ([20, Theorem 2.3.8]) and by the embedding $L^{\infty} \hookrightarrow$ $B_{\infty, \infty}^{0}$, we have

$$
\left\|m_{j}\right\|_{B_{\infty, \infty}^{N}} \approx \sum_{|\gamma| \leq N}\left\|\partial^{\gamma} m_{j}\right\|_{B_{\infty, \infty}^{0}} \lesssim \sum_{|\gamma| \leq N}\left\|\partial^{\gamma} m_{j}\right\|_{L^{\infty}}
$$

Since $\Psi$ appearing in (1.3) satisfies supp $\Psi \subset\{1 / 2 \leq|\zeta| \leq 2\}$, we see that

$$
\begin{aligned}
\left|\partial^{\gamma} m_{j}(\zeta)\right| & =\left|\sum_{\gamma_{1}+\gamma_{2}=\gamma} C_{\gamma_{1}, \gamma_{2}} 2^{j\left|\gamma_{1}\right|}\left(\partial^{\gamma_{1}} m\right)\left(2^{j} \zeta\right)\left(\partial^{\gamma_{2}} \Psi\right)(\zeta)\right| \\
& \lesssim \sum_{\gamma_{1}+\gamma_{2}=\gamma} 2^{j\left|\gamma_{1}\right|} A\left(1+2^{j}|\zeta|\right)^{-s}\left|\left(\partial^{\gamma_{2}} \Psi\right)(\zeta)\right| \lesssim A\left(\max \left\{1,2^{j}\right\}\right)^{|\gamma|-s}
\end{aligned}
$$

for $|\gamma| \leq N$. Hence

$$
\left\|m_{j}\right\|_{B_{\infty}^{N}, \infty} \lesssim \sum_{|\gamma| \leq N} A\left(\max \left\{1,2^{j}\right\}\right)^{|\gamma|-s} \approx A\left(\max \left\{1,2^{j}\right\}\right)^{N-s} .
$$

Similarly we have

$$
\left\|m_{j}\right\|_{B_{\infty, \infty}^{0}} \lesssim\left\|m_{j}\right\|_{L^{\infty}} \lesssim A\left(\max \left\{1,2^{j}\right\}\right)^{-s}
$$

Since $s=\theta N$, combining (4.8), (4.10), and (4.11), we have

$$
\left\|m_{j}\right\|_{B_{\infty}^{s}, \infty} \lesssim\left\{A\left(\max \left\{1,2^{j}\right\}\right)^{-s}\right\}^{(1-\theta)}\left\{A\left(\max \left\{1,2^{j}\right\}\right)^{N-s}\right\}^{\theta}=A .
$$

This completes the proof.

PROOF OF THE LATTER PART OF THEOREM 1.2. Let $p, q, r$ satisfy the assumptions of Theorem 1.2 and let $s(p, q)$ be the number as given in the theorem. Assume that $s>0$ and $t \in[1, \infty]$ satisfy

$$
\left\|T_{m}\right\|_{H^{p} \times H^{q} \rightarrow L^{r}} \lesssim \sup _{j \in \boldsymbol{Z}}\left\|m_{j}\right\|_{B_{t, \infty}^{s}}
$$

where $H^{\infty} \times H^{\infty} \rightarrow L^{\infty}$ is replaced by $L^{\infty} \times L^{\infty} \rightarrow B M O$ in the case $p=q=r=\infty$. Taking $\widetilde{\Psi} \in \mathcal{S}\left(\boldsymbol{R}^{2 n}\right)$ satisfying $\widetilde{\Psi}=1$ on supp $\Psi$, where $\Psi$ is the function appearing in (1.3), we can write $m_{j}(\zeta)=\widetilde{\Psi}(\zeta) m_{j}(\zeta)$. By Lemma 2.3,

$$
\left\|m_{j}\right\|_{B_{t, \infty}^{s}} \lesssim\|\widetilde{\Psi}\|_{B_{t, \infty}^{s}}\left\|m_{j}\right\|_{B_{\infty, \infty}^{s}} \approx\left\|m_{j}\right\|_{B_{\infty, \infty}^{s}} .
$$

From (4.12), (4.13), and Lemma 4.2, we have

$$
\left\|T_{m}\right\|_{H^{p} \times H^{q} \rightarrow L^{r}} \lesssim \sup _{j \in \boldsymbol{Z}}\left\|m_{j}\right\|_{B_{\infty, \infty}^{s}} \lesssim \max _{|\alpha|+|\beta| \leq[s]+1}\left(\sup _{\xi, \eta \in \boldsymbol{R}^{n}}(1+|\xi|+|\eta|)^{s}\left|\partial_{\xi}^{\alpha} \partial_{\eta}^{\beta} m(\xi, \eta)\right|\right) .
$$

Hence we must have $s \geq s(p, q)$ by Theorem B. This completes the proof. 
5. A comparison of Theorem 1.2 and Theorem A. The following proposition shows that the case $s(p, q)<n$ of Theorem 1.2 covers multipliers that cannot be dealt with by Theorem A.

Proposition 5.1. Let $0<s<n, s_{1}>n / 2$, and $s_{2}>n / 2$. Then there exists $a$ function $m(\xi, \eta)$ on $\boldsymbol{R}^{n} \times \boldsymbol{R}^{n}$ such that $\sup _{j \in \boldsymbol{Z}}\left\|m_{j}\right\|_{B_{t, \infty}^{s}\left(\boldsymbol{R}^{2 n}\right)}<\infty$ for any $1 \leq t \leq \infty$, but $\sup _{j \in \boldsymbol{Z}}\left\|m_{j}\right\|_{W^{\left(s_{1}, s_{2}\right)}\left(\boldsymbol{R}^{2 n)}\right)}=\infty$, where $m_{j}$ is defined by (1.3).

PROOF. We shall divide the proof into three steps. In Steps 1 and 2, we assume that $m(\zeta)$ is a function in $C^{\infty}\left(\boldsymbol{R}^{2 n}\right)$ satisfying supp $m \subset\{|\zeta| \geq 1\}$, where $\zeta=(\xi, \eta) \in \boldsymbol{R}^{n} \times \boldsymbol{R}^{n}$.

Step 1. Let $s>0$. In this step, we prove that if $m(\zeta)$ satisfies

$$
\left|\partial_{\zeta}^{\gamma} m(\zeta)\right| \leq C_{\gamma}|\zeta|^{-s}, \quad \gamma \in N_{0}^{2 n}
$$

then $\sup _{j \in \boldsymbol{Z}}\left\|m_{j}\right\|_{B_{t, \infty}^{s}}<\infty$ for any $1 \leq t \leq \infty$. By (4.13), it is sufficient to prove $\sup _{j \in \boldsymbol{Z}}\left\|m_{j}\right\|_{B_{\infty, \infty}^{s}}<\infty$ under the assumption (5.1). This follows from Lemma 4.2, since $m(\zeta)$ satisfies

$$
\left|\partial_{\zeta}^{\gamma} m(\zeta)\right| \leq C_{\gamma}(1+|\zeta|)^{-s}, \quad \gamma \in N_{0}^{2 n}
$$

where we have used the fact $\operatorname{supp} m \subset\{|\zeta| \geq 1\}$.

Step 2. Let $s_{1}>n / 2$ and $s_{2}>n / 2$. In this step, we prove that if $m(\zeta)$ satisfies $\sup _{j \in \boldsymbol{Z}}\left\|m_{j}\right\|_{W^{\left(s_{1}, s_{2}\right)}}<\infty$, then for any $\varepsilon>0$

$$
\mathcal{F}^{-1}\left[|\zeta|^{-\varepsilon} m(\zeta)\right] \in L^{1}\left(\boldsymbol{R}^{2 n}\right) \text {. }
$$

Taking $\widetilde{\Psi} \in \mathcal{S}\left(\boldsymbol{R}^{2 n}\right)$ satisfying $\widetilde{\Psi}=1$ on $\operatorname{supp} \Psi$ and $\operatorname{supp} \widetilde{\Psi} \not \supset 0$, where $\Psi$ is the function appearing in (1.3), we can write $|\zeta|^{-\varepsilon} m_{j}\left(\zeta / 2^{j}\right)=|\zeta|^{-\varepsilon} \widetilde{\Psi}\left(\zeta / 2^{j}\right) m_{j}\left(\zeta / 2^{j}\right)$. Then, by Young's inequality and changes of variables,

$$
\begin{aligned}
\left\|\mathcal{F}^{-1}\left[|\cdot|^{-\varepsilon} m_{j}\left(\cdot / 2^{j}\right)\right]\right\|_{L^{1}} & \leq\left\|\mathcal{F}^{-1}\left[|\cdot|^{-\varepsilon} \widetilde{\Psi}\left(\cdot / 2^{j}\right)\right]\right\|_{L^{1}}\left\|\mathcal{F}^{-1}\left[m_{j}\left(\cdot / 2^{j}\right)\right]\right\|_{L^{1}} \\
& =2^{-j \varepsilon}\left\|\mathcal{F}^{-1}\left[|\cdot|^{-\varepsilon} \widetilde{\Psi}\right]\right\|_{L^{1}}\left\|\mathcal{F}^{-1} m_{j}\right\|_{L^{1}}=C 2^{-j \varepsilon}\left\|\mathcal{F}^{-1} m_{j}\right\|_{L^{1}} .
\end{aligned}
$$

Since $s_{1}, s_{2}>n / 2$, we have by Schwarz's inequality and Plancherel's theorem

$$
\begin{aligned}
\left\|\mathcal{F}^{-1} m_{j}\right\|_{L^{1}}= & \frac{1}{(2 \pi)^{2 n}} \int_{\boldsymbol{R}^{n}} \int_{\boldsymbol{R}^{n}}\left|\widehat{m_{j}}(x, y)\right| d x d y \\
= & \frac{1}{(2 \pi)^{2 n}} \int_{\boldsymbol{R}^{n}} \int_{\boldsymbol{R}^{n}} \frac{\left(1+|x|^{2}\right)^{s_{1} / 2}\left(1+|y|^{2}\right)^{s_{2} / 2}\left|\widehat{m_{j}}(x, y)\right|}{\left(1+|x|^{2}\right)^{s_{1} / 2}\left(1+|y|^{2}\right)^{s_{2} / 2}} d x d y \\
\lesssim & \left(\int_{\boldsymbol{R}^{n}} \int_{\boldsymbol{R}^{n}} \frac{1}{\left(1+|x|^{2}\right)^{s_{1}}\left(1+|y|^{2}\right)^{s_{2}}} d x d y\right)^{1 / 2} \\
& \times\left(\int_{\boldsymbol{R}^{n}} \int_{\boldsymbol{R}^{n}}\left|\left(1+|x|^{2}\right)^{s_{1} / 2}\left(1+|y|^{2}\right)^{s_{2} / 2} \widehat{m_{j}}(x, y)\right|^{2} d x d y\right)^{1 / 2} \\
= & C\left\|\left(I-\Delta_{\xi}\right)^{s_{1} / 2}\left(I-\Delta_{\eta}\right)^{s_{2} / 2} m_{j}(\xi, \eta)\right\|_{L^{2}}=C\left\|m_{j}\right\|_{W^{\left(s_{1}, s_{2}\right)}} \lesssim 1,
\end{aligned}
$$

and consequently

$$
\left\|\mathcal{F}^{-1}\left[|\cdot|^{-\varepsilon} m_{j}\left(\cdot / 2^{j}\right)\right]\right\|_{L^{1}} \lesssim 2^{-j \varepsilon}
$$


Hence, using the decomposition

$$
|\zeta|^{-\varepsilon} m(\zeta)=\sum_{j=0}^{\infty}|\zeta|^{-\varepsilon} m(\zeta) \Psi\left(\zeta / 2^{j}\right)=\sum_{j=0}^{\infty}|\zeta|^{-\varepsilon} m_{j}\left(\zeta / 2^{j}\right)
$$

where we have used the facts that $\operatorname{supp} m \subset\{|\zeta| \geq 1\}$ and $\operatorname{supp} \Psi\left(\cdot / 2^{j}\right) \subset\left\{2^{j-1} \leq|\zeta| \leq\right.$ $2^{j+1}$, we have

$$
\left\|\mathcal{F}^{-1}\left[|\cdot|^{-\varepsilon} m\right]\right\|_{L^{1}} \leq \sum_{j=0}^{\infty}\left\|\mathcal{F}^{-1}\left[|\cdot|^{-\varepsilon} m_{j}\left(\cdot / 2^{j}\right)\right]\right\|_{L^{1}} \lesssim \sum_{j=0}^{\infty} 2^{-j \varepsilon}<\infty .
$$

Step 3. In this step, we finish the proof of Proposition 5.1. We assume that $0<s<n$, $s_{1}>n / 2$, and $s_{2}>n / 2$. Take a number $0<a<1$ satisfying $s<n a$. Let us consider the function $m(\zeta)$ defined by

$$
m(\zeta)=\Theta(\zeta)|\zeta|^{-s} \exp \left(i|\zeta|^{a}\right), \quad \zeta \in \boldsymbol{R}^{2 n}
$$

where $\Theta \in C^{\infty}\left(\boldsymbol{R}^{2 n}\right)$ satisfies $\Theta(\zeta)=0$ if $|\zeta| \leq 1$ and $\Theta(\zeta)=1$ if $|\zeta| \geq 2$. We shall see that this $m(\zeta)$ is a function which we are looking for.

It is easy to check that $m(\zeta)$ defined by (5.3) satisfies (5.1). Thus, it follows from Step 1 that $\sup _{j \in \boldsymbol{Z}}\left\|m_{j}\right\|_{B_{t, \infty}^{s}}<\infty$ for any $1 \leq t \leq \infty$. On the other hand, by [21, Theorem 9], if $s+\varepsilon \leq 2 n-n a$, then

$$
\left|\mathcal{F}^{-1}\left[|\cdot|^{-\varepsilon} m\right](X)\right| \approx|X|^{(-2 n+s+\varepsilon+n a) /(1-a)}, \quad X \in \boldsymbol{R}^{2 n}, \quad X \rightarrow 0 .
$$

From this we see that $\mathcal{F}^{-1}\left[|\cdot|^{-\varepsilon} m\right] \in L^{1}\left(\boldsymbol{R}^{2 n}\right)$ if and only if $s+\varepsilon>n a$. Hence, if $\varepsilon>0$ is so small that $s+\varepsilon \leq n a$ (this is possible because $s<n a$ ), then $\mathcal{F}^{-1}\left[|\cdot|^{-\varepsilon} m\right] \notin L^{1}\left(\boldsymbol{R}^{2 n}\right)$, namely (5.2) does not hold for such small $\varepsilon$. Therefore, it follows from Step 2 that $m(\zeta)$ does not satisfy $\sup _{j \in \boldsymbol{Z}}\left\|m_{j}\right\|_{W^{\left(s_{1}, s_{2}\right)}}<\infty$. The proof is complete.

Appendix A. The first commutator of Calderón is defined by

$$
\mathcal{C}_{1}(f)(x)=\text { p.v. } \int_{\boldsymbol{R}} \frac{A(x)-A(y)}{(x-y)^{2}} f(y) d y .
$$

It is known that $\mathcal{C}_{1}$ is a bounded operator in $L^{p}(\boldsymbol{R}), 1<p<\infty$, if $A$ is a Lipschitz function on $\boldsymbol{R}$ and

$$
\left\|\mathcal{C}_{1}(f)\right\|_{L^{p}(\boldsymbol{R})} \leq c_{p}\left\|A^{\prime}\right\|_{L^{\infty}(\boldsymbol{R})}\|f\|_{L^{p}(\boldsymbol{R})}, \quad 1<p<\infty .
$$

See Calderón [1], Coifman-McIntosh-Meyer [2] and Coifman-Meyer [12] for its history. In this appendix, we shall observe that the estimate (A.1) and other estimates for the case $A^{\prime} \in$ $L^{q}(\boldsymbol{R})$ immediately follow from Theorem 1.2.

If we set $A^{\prime}=a$, then $\mathcal{C}_{1}$ can be written as a bilinear Fourier multiplier operator concerning $f$ and $a$ :

$$
\mathcal{C}_{1}(f, a)(x)=\frac{1}{(2 \pi)^{2}} \int_{\boldsymbol{R}^{2}} e^{i x(\xi+\eta)}\left(-i \pi \int_{0}^{1} \operatorname{sgn}(\xi+\alpha \eta) d \alpha\right) \widehat{f}(\xi) \widehat{a}(\eta) d \xi d \eta .
$$


Since

$$
\int_{0}^{1} \operatorname{sgn}(\xi+\alpha \eta) d \alpha=2 \int_{0}^{1} \chi_{(0, \infty)}(\xi+\alpha \eta) d \alpha-1,
$$

we can reduce the boundedness of $\mathcal{C}_{1}$ to that of $T_{m}$ with

$$
m(\xi, \eta)=\int_{0}^{1} \chi_{(0, \infty)}(\xi+\alpha \eta) d \alpha, \quad(\xi, \eta) \in \boldsymbol{R} \times \boldsymbol{R} .
$$

This explanation can be found in Muscalu [15].

Suppose $1<p, q \leq \infty, 1 / p+1 / q=1 / r$, and $2 / 3<r<\infty$. Let $s(p, q)$ and $t(p, q)$ be the numbers given in Theorem 1.2. Note that we are treating the case $n=1$ and observe that $s(p, q)<1$. Hence Theorem 1.2 and (4.13) yield

$$
\left\|T_{m}\right\|_{L^{p} \times L^{q} \rightarrow L^{r}} \lesssim \sup _{j \in \boldsymbol{Z}}\left\|m_{j}\right\|_{B_{\infty, \infty}^{1}}
$$

Thus, once the condition

$$
\sup _{j \in \boldsymbol{Z}}\left\|m_{j}\right\|_{B_{\infty, \infty}^{1}}<\infty
$$

is checked, we obtain

$$
\left\|\mathcal{C}_{1}(f, a)\right\|_{L^{r}} \lesssim\|f\|_{L^{p}}\|a\|_{L^{q}}
$$

the estimate (A.1) is the case $q=\infty$ of this estimate.

Since $m(\xi, \eta)$ defined by (A.2) is homogeneous of degree 0 , to obtain (A.3), it is sufficient to consider the case $j=0$, namely $m_{0}(\xi, \eta)=m(\xi, \eta) \Psi(\xi, \eta)$. Hence, by (4.9), our goal is to check

$$
\left\|m_{0}(\xi, \eta)\right\|_{L^{\infty}}+\left\|\partial_{\xi} m_{0}(\xi, \eta)\right\|_{L^{\infty}}+\left\|\partial_{\eta} m_{0}(\xi, \eta)\right\|_{L^{\infty}}<\infty .
$$

Note that $m(\xi, \eta)$ can be written as

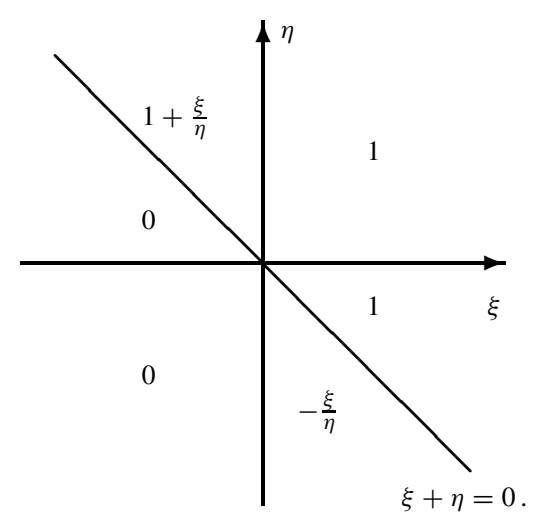

Since the function $m(\xi, \eta)$ is Lipschitz continuous away from the origin, the estimate (A.4) is obvious. 


\section{REFERENCES}

[1] A. CAlderón, Commutators of singular integral operators, Proc. Nat. Acad. Sci. U.S.A. 53 (1965), 10921099.

[2] R. Coifman, A. Mcintosh And Y. Meyer, L'intégrale de Cauchy définit un opérateur borné sur $L^{2}$ pour les courbes lipschitziennes, Ann. of Math. 116 (1982), 361-387.

[3] R. CoIfman AND Y. MEYER, Nonlinear harmonic analysis, operator theory and P.D.E., in Beijing lectures in harmonic analysis (Beijing, 1984), 3-45, Ann. of Math. Stud. 112, Princeton Univ. Press, Princeton, NJ, 1986.

[4] M. Fujita And N. Tomita, Weighted norm inequalities for multilinear Fourier multipliers, Trans. Amer. Math. Soc. 364 (2012), 6335-6353.

[5] L. Grafakos And N. Kalton, Multilinear Calderón-Zygmund operators on Hardy spaces, Collect. Math. 52 (2001), 169-179.

[6] L. Grafakos, A. Miyachi and N. Tomita, On multilinear Fourier multipliers of limited smoothness, Canad. J. Math. 65 (2013), 299-330.

[7] L. GRAFAKos AND Z. Si, The Hörmander multiplier theorem for multilinear operators, J. Reine Angew. Math. 668 (2012), 133-147.

[8] L. GRAFAKos AND R. TORRES, Discrete decompositions for bilinear operators and almost diagonal conditions, Trans. Amer. Math. Soc. 354 (2002), 1153-1176.

[9] L. Grafakos And R. Torres, Multilinear Calderón-Zygmund theory, Adv. Math. 165 (2002), 124-164.

[10] S. JANSON AND P. W. Jones, Interpolation between $H^{p}$ spaces: the complex method, J. Funct. Anal. 48 (1982), 58-80.

[11] C. Kenig And E. M. Stein, Multilinear estimates and fractional integration, Math. Res. Lett. 6 (1999), 1-15.

[12] Y. Meyer and R. Coifman, Wavelets: Calderón-Zygmund and multilinear operators, Cambridge Stud. Adv. Math. 48, Cambridge University Press, Cambridge, 1997.

[13] A. Miyachi And N. Tomita, Minimal smoothness conditions for bilinear Fourier multipliers, Rev. Mat. Iberoam. 29 (2013), 495-530.

[14] A. MiYachi AND N. TomitA, Calderón-Vaillancourt type theorem for bilinear pseudo-differential operators, Indiana Univ. Math. J., to appear.

[15] C. Muscalu, Calderón commutators and the Cauchy integral on Lipschitz curves revisited I. First commutator and generalizations, arXiv:1201.3845, 2012.

[16] T. RUNST AND W. SickeL, Sobolev spaces of fractional order, Nemytskij operators, and nonlinear partial differential equations, De Gruyter Ser. Nonlinear Anal. Appl. 3, Walter de Gruyter \& Co., Berlin, 1996.

[17] E. M. SteIn, Harmonic analysis: real-variable methods, orthogonality, and oscillatory integrals, Princeton Math. Ser. 43, Princeton University Press, Princeton, 1993.

[18] E. M. STEIn AND G. Weiss, On the interpolation of analytic families of operators acting on $H^{p}$-spaces, Tôhoku Math. J. 9 (1957), 318-339.

[19] N. Tomita, A Hörmander type multiplier theorem for multilinear operators, J. Funct. Anal. 259 (2010), 2028-2044.

[20] H. TRIEBEL, Theory of Function Spaces, Birkhäuser Verlag, Basel, 1983.

[21] S. Wainger, Special trigonometric series in $k$-dimensions, Mem. Amer. Math. Soc. No. 59, 1965.

\author{
DEPARTMENT OF MATHEMATICS \\ TOKYO WOMAN'S CHRISTIAN UNIVERSITY \\ ZEMPUKUJI, SUGINAMI-KU \\ TOKYO 167-8585 \\ JAPAN \\ E-mail address: miyachi@lab.twcu.ac.jp
}

\author{
DEPARTMENT OF MATHEMATICS \\ OSAKA UNIVERSITY \\ TOYONAKA \\ OSAKA 560-0043 \\ JAPAN \\ E-mail address: tomita@math.sci.osaka-u.ac.jp
}

